CONFORMAL GEOMETRY AND DYNAMICS

An Electronic Journal of the American Mathematical Society

Volume 4, Pages 75-96 (April 27, 2000)

S $1088-4173(00) 00056-4$

\title{
A COMBINATION THEOREM FOR COVERING CORRESPONDENCES AND AN APPLICATION TO MATING POLYNOMIAL MAPS WITH KLEINIAN GROUPS
}

\author{
SHAUN BULLETT
}

\begin{abstract}
The simplest version of the Maskit-Klein combination theorems concerns the action of a free product of two finite subgroups of $\operatorname{PSL}(2, \mathbb{C})$ on the Riemann sphere $\hat{\mathbb{C}}$, when these subgroups have fundamental domains whose interiors together cover $\hat{\mathbb{C}}$. We prove an analogous combination theorem for covering correspondences of rational maps, making use of Douady and Hubbard's Straightening Theorem for polynomial-like maps to describe the structure of the limit sets. We apply our theorem to construct holomorphic correspondences which are matings of polynomial maps with Hecke groups $C_{p} * C_{q}$, and we show how it may also be applied to the analysis of separable correspondences.
\end{abstract}

\section{COVERING CORRESPONDENCES AND TRANSVERSAlS FOR RATIONAL MAPS}

For any rational map $Q: \hat{\mathbb{C}} \rightarrow \hat{\mathbb{C}}$ of degree $q$ we define

- $\operatorname{Cov}^{Q}$ to be the $(q: q)$ correspondence (multi-valued map) $z \rightarrow w$ where $w$ runs through all values such that

$$
Q(w)=Q(z)
$$

and

- $\mathrm{Gal}^{Q}$ to be the $(q-1: q-1)$ correspondence $z \rightarrow w$ where

$$
\frac{Q(w)-Q(z)}{w-z}=0
$$

We shall use the same notation for both the graph of a correspondence and the multi-valued map defined by that graph. Thus $\operatorname{Cov}^{Q}=\operatorname{Gal}^{Q} \cup I$, where $I$ denotes the graph of the identity. The intersection $\operatorname{Gal}^{Q} \cap I$ consists precisely of the points $(z, z)$ such that $z$ is a critical point of $Q$. Recall that $Q$ has $2 q-2$ critical points, counted with multiplicity. The reader should be warned of a small change in terminology from our earlier usage [1, 2, 3] in which $\mathrm{Gal}^{Q}$ was taken to be the correspondence sending $z$ to all $w \neq z$ satisfying $Q(w)=Q(z)$. Our new $G_{a l}{ }^{Q}$ has graph the closure of that given by the old definition.

In general, by a holomorphic correspondence on $\hat{\mathbb{C}}$ we shall mean any multivalued map $z \rightarrow w$ defined by a polynomial relation $p(z, w)=0$. Examples are rational maps $((n: 1)$ correspondences $)$, their inverses $((1: n)$ correspondences $)$,

Received by the editors September 30, 1999 and, in revised form, January 20, 2000. 2000 Mathematics Subject Classification. Primary 37F05; Secondary 30D05, 30F40.

Key words and phrases. Holomorphic dynamics, polynomial maps, Kleinian groups.

I would like to thank Christopher Penrose for many helpful discussions concerning this work. 


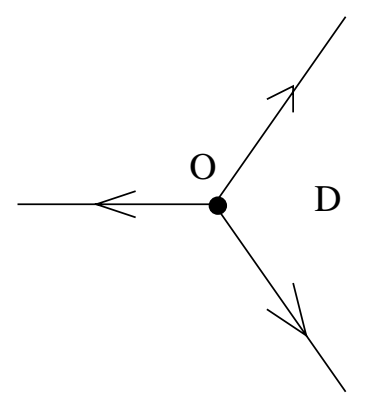

FiguRE 1.

and $n$-generator subgroups of $\operatorname{PSL}(2, \mathbb{C})((n: n)$ correspondences). Covering correspondences of rational maps are a natural generalisation of finite subgroups of $P S L(2, \mathbb{C})$.

1.1. Example. $Q(z)=z^{3}$. This function has double critical points at 0 and $\infty$. Here

$$
\operatorname{Cov}^{Q}: z \rightarrow\left\{z, e^{2 \pi i / 3} z, e^{4 \pi i / 3} z\right\}
$$

and

$$
G_{a l}^{Q}: z \rightarrow\left\{e^{2 \pi i / 3} z, e^{4 \pi i / 3} z\right\}
$$

Thus

$$
\operatorname{Cov}^{Q}=\left\{I, \rho, \rho^{2}\right\}=C_{3},
$$

the cyclic group of order 3 generated by $\rho: z \rightarrow e^{2 \pi i / 3} z$, and $G a l^{Q}$ consists of the non-identity elements $\left\{\rho, \rho^{2}\right\}$. A fundamental domain $D$ for the action of $\rho$ on the Riemann sphere $\hat{\mathbb{C}}$ is illustrated in Figure 1. We write $\operatorname{Gal}^{Q}(D)$ for the set of all images $w$ of points $z \in D$ under $G a l^{Q}$. Different authors have slightly different notions of a 'fundamental domain' but note that whatever definition is chosen the interiors of $D$ and $\operatorname{Gal}^{Q}(D)$ are disjoint and their closures together cover $\hat{\mathbb{C}}$.

1.2. Example. $Q(z)=z^{3}-3 z$. This has a double critical point at $\infty$ and simple critical points at \pm 1 . Now

$$
\operatorname{Cov}^{Q}: z \rightarrow w \text { where } w^{3}-3 w=z^{3}-3 z
$$

and

$$
G a l^{Q}: z \rightarrow w \text { where } w^{2}+w z+z^{2}=3 .
$$

There is no longer an action of the cyclic group of order 3 , but there is an analogue of a fundamental domain, namely any transversal for $Q: \hat{\mathbb{C}} \rightarrow \hat{\mathbb{C}}$, that is to say any maximal subset of $\hat{\mathbb{C}}$ on which $Q$ is injective. An example of such a transversal $D_{Q}$ is illustrated in Figure 2. Here $D_{Q}$ is the open right hand region together with the part of its boundary lying in the upper half-plane (the curve running from 1 to $\infty$, including both of these points).

Note that for any rational map $Q$ and transversal $D_{Q}$ the sets $D_{Q}$ and $G a l^{Q}\left(D_{Q}\right)$ together cover $\hat{\mathbb{C}}$ and their intersection is the set of critical points of $Q$ which lie in $D_{Q}$ (necessarily on the boundary). In our example the critical point 1 lies in $D_{Q}$ : its images under $G a l^{Q}$ are itself and the corresponding co-critical point -2 


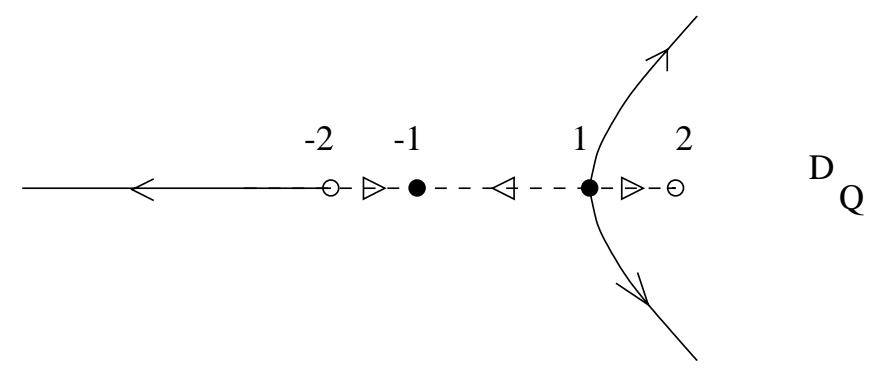

FIGURE 2 .

(the distinct image of 1 under $G a l^{Q}$ ). The critical point $\infty$ also lies in $D_{Q}$, but has only one image under $G a l^{Q}$, namely itself. The critical point -1 lies outside $D_{Q}$ : its images under $\mathrm{Gal}^{Q}$ are itself and the corresponding co-critical point 2 (which lies in the interior of $\left.D_{Q}\right)$. In order to divide $\operatorname{Gal}^{Q}\left(D_{Q}\right)$ in this example into two 'tiles', each mapping bijectively onto $D_{Q}$ but allowed to intersect at critical points, we must choose a curve along which to cut between the critical points -1 and 1 : the part of the tile boundary from -1 to -2 is an image of this curve under $\mathrm{Gal}^{Q}$.

Up to pre- and post-composition by Möbius transformations every degree 3 rational function with exactly two distinct critical points is equivalent to Example 1.1, and every degree 3 rational function with exactly three distinct critical points is equivalent to Example 1.2. Any other degree 3 rational function has four distinct critical points: its equivalence class is determined by their cross-ratio.

Lemma 1. Let $D_{Q}$ be a transversal of a rational map $Q$ on $\hat{\mathbb{C}}$ and let $D_{Q}^{\circ}$ and $\overline{D_{Q}}$ denote the interior and closure of $D_{Q}$ respectively. Then

(i) the restriction of $\mathrm{Gal}^{Q}$ to domain $\mathrm{Gal}^{Q}\left(D_{Q}\right)$ and range $D_{Q}$ is a single-valued (though not in general continuous) surjection;

(ii) $G_{a l}^{Q}\left(D_{Q}^{\circ}\right)$ is open and $G a l^{Q}: G a l^{Q}\left(D_{Q}^{\circ}\right) \rightarrow D_{Q}^{\circ}$ is a complex analytic map of degree $q-1$ (where $q$ is the degree of $Q$ );

(iii) if $\overline{D_{Q}^{\circ}}=\overline{D_{Q}}$, then $\operatorname{Gal}^{Q}\left(D_{Q}^{\circ}\right)$ is disjoint from $\overline{D_{Q}}$.

Proof. Part (i) follows at once from the definition of a transversal. For part (ii) observe that $D_{Q}^{\circ}$ cannot contain any critical point of $Q$ and thus every point $z \in D_{Q}^{\circ}$ has an open disc neighbourhood $U$ such that $\mathrm{Gal}^{Q}(U)$ is a disjoint union of open discs, together branch-covering $U$ with degree $(k-1)$ under the complex analytic map $G a l^{Q}: G a l^{Q}(U) \rightarrow U$, with ramification points at critical points of $Q$. Thus $\operatorname{Gal}^{Q}\left(D_{Q}^{\circ}\right)$ is open and $\operatorname{Gal}^{Q}: G a l^{Q}\left(D_{Q}^{\circ}\right) \rightarrow D_{Q}^{\circ}$ is complex analytic. For (iii) observe that $G a l^{Q}\left(D_{Q}^{\circ}\right)$ is disjoint from $D_{Q}^{\circ}$ (since $D_{Q}$ is a transversal), and as it is open it is disjoint from $\overline{D_{Q}^{\circ}}=\overline{D_{Q}}$.

We remark that $G a l^{Q}: G a l^{Q}\left(D_{Q}\right) \rightarrow D_{Q}$ is strictly $(k-1)$-to-one except over cocritical points of $Q$ in $D_{Q}$ (where the notion of co-critical point is taken to include any critical points of $Q$ of multiplicity $>1$ ). In the case illustrated in Figure 2, $\operatorname{Gal}^{Q}\left(D_{Q}\right)=\left(\hat{\mathbb{C}}-D_{Q}\right) \cup\{1\} \cup\{\infty\}$ and the single-valued map $\operatorname{Gal}^{Q}\left(D_{Q}\right) \rightarrow D_{Q}$ defined by $\mathrm{Gal}^{Q}$ is two-to-one except over $\infty$ and 2. This map is continuous except on $(-\infty,-2) \subset \mathbb{R}$. The set $\operatorname{Gal}^{Q}\left(D_{Q}^{\circ}\right)$ is the region $\hat{\mathbb{C}}-\overline{D_{Q}}$ with $[-\infty,-2]$ removed, and restricted to $G a l^{Q}\left(D_{Q}^{\circ}\right)$ the map defined by $G a l^{Q}$ is complex analytic. 


\section{KLEIN'S COMBINATION THEOREM FOR GROUPS}

The basic situation for Klein's combination theorem [7] is that of a pair of finitely generated discrete subgroups $H$ and $K$ of $P S L(2, \mathbb{C})$ having fundamental domains $D_{H}$ and $D_{K}$ with interiors together covering the Riemann sphere $\hat{\mathbb{C}}$. For our generalisation to correspondences we shall only need to consider finite subgroups $H$ and $K$ of $P S L(2, \mathbb{C})$, which of course are necessarily discrete. We start by defining our terms.

Definitions. Let $G$ be any finitely generated Kleinian group, that is to say any finitely generated discrete subgroup of $P S L(2, \mathbb{C})$.

- The ordinary set (or discontinuity set) $\Omega(G)$ of $G$ is the set of all points $z \in \hat{\mathbb{C}}$ having a neighbourhood $U$ such that $U \cap g(U) \neq \emptyset$ for only finitely many $g \in G$.

- The free ordinary set $\Omega^{\prime}(G)$ is the subset of $\Omega(G)$ of points $z$ having a neighbourhood $U$ such that $U \cap g(U)=\emptyset$ for all $g \in G-\{I\}$. (The free ordinary set is obtained from the ordinary set by puncturing it at the discrete set of points which have non-trivial stabiliser.)

- The limit set $\Lambda(G)$ is the set of all points $z$ such that there exists $z_{0} \in \hat{\mathbb{C}}$ and distinct $g_{n} \in G$ with $\lim _{n \rightarrow \infty} g_{n}\left(z_{0}\right)=z$. (The limit set is the complement in $\hat{\mathbb{C}}$ of the ordinary set.)

- A transversal $T$ for the action of $G$ on $\Omega=\Omega(G)$ is a maximal subset of $\Omega$ on which the orbit quotient map $\Omega \rightarrow \Omega / G$ is injective.

- A fundamental set $S$ for the action of $G$ on $\Omega^{\prime}=\Omega^{\prime}(G)$ is a subset $S$ of $\Omega^{\prime}$ such that $g(S) \cap h(S)=\emptyset$ when $g$ and $h$ are any two distinct elements of $G$, and $\bigcup_{g \in G} g(S)=\Omega^{\prime}$. (Equivalently $S$ is a transversal for the action of $G$ on $\Omega^{\prime}$.)

In Example 1.1 above the ordinary set $\Omega$ for the action of $C_{3}$ is the whole of $\hat{\mathbb{C}}$ and the free ordinary set $\Omega^{\prime}$ is $\hat{\mathbb{C}}-\{0, \infty\}$. For the set $D$ illustrated in Figure 1 to be a transversal for the action of $G$ on $\Omega$ it must contain both 0 and $\infty$, and exactly one out of each pair of points identified under $C_{3}$ on the remaining part of the boundary. To make it into a fundamental set $D^{\prime}$ for the action of $C_{3}$ on $\Omega^{\prime}$ we excise 0 and $\infty$.

Theorem 1. Let $H$ and $K$ be non-trivial finite subgroups of $P S L(2, \mathbb{C})$, let $D_{H}$ and $D_{K}$ be transversals for the actions of $H$ and $K$ respectively on $\hat{\mathbb{C}}$, having interiors $D_{H}^{\circ}, D_{K}^{\circ}$ with $\overline{D_{H}^{\circ}}=\overline{D_{H}}$ and $\overline{D_{K}^{\circ}}=\overline{D_{K}}$, and suppose that $D_{H}^{\circ} \cup D_{K}^{\circ}=\hat{\mathbb{C}}$. Let $D_{H}^{\prime}$ and $D_{K}^{\prime}$ denote the fundamental sets for the actions of $H$ and $K$ on $\Omega^{\prime}(H)$ and $\Omega^{\prime}(K)$ obtained by removing from $D_{H}$ and $D_{K}$ those points which have non-trivial stabilisers under the respective groups. Let $\Delta$ denote $D_{H} \cap D_{K}$ and $\Delta^{\prime}$ denote $D_{H}^{\prime} \cap D_{K}^{\prime}$. Then:

- The free product $G=H * K$ acts freely on $\Omega\left(G, \Delta^{\prime}\right)=\bigcup_{g \in G} g\left(\Delta^{\prime}\right)$, and $\Delta^{\prime}$ is a fundamental set for this action.

- $\Omega\left(G, \Delta^{\prime}\right)=\Omega^{\prime}(G)$, the free ordinary set for the action of $G$ on $\hat{\mathbb{C}}$.

- $G$ acts properly discontinuously on $\Omega(G, \Delta)=\bigcup_{g \in G} g(\Delta)$, and $\Delta$ is a transversal for this action.

- $\Omega(G, \Delta)=\Omega(G)$, the ordinary set for the action of $G$ on $\hat{\mathbb{C}}$.

- $\Lambda(G)(=\hat{\mathbb{C}}-\Omega(G, \Delta))$ is a Cantor set except in the special case that $H$ and $K$ are both cyclic of order 2 , in which case $\Lambda(G)$ consists of exactly two points.

This is essentially the classical theorem of Klein [7], in the special case of finite groups. Proofs of one form or another of this theorem can be found in standard 

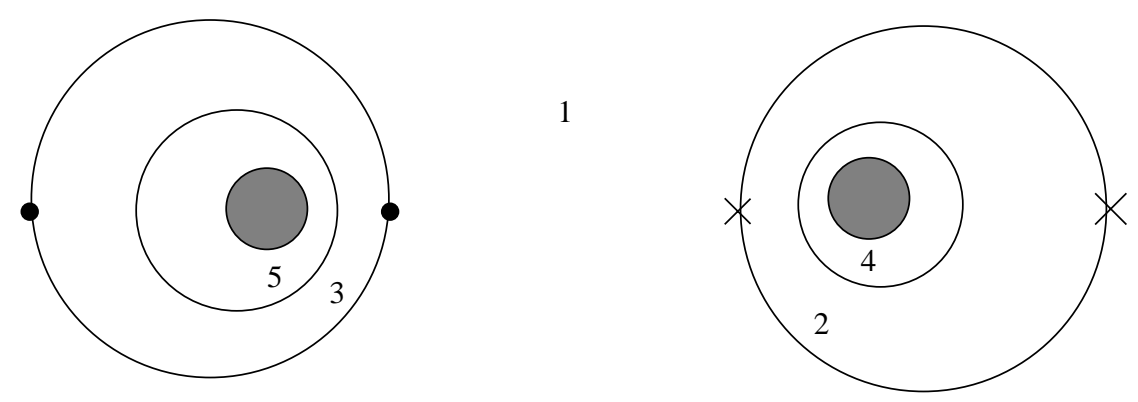

FIGURE 3.

texts on Kleinian groups. The exact wording above is motivated by the analogous statement for covering correspondences of rational maps which we shall prove in the next section, and which contains Theorem 1 as a particular family of cases. More general combination theorems for Kleinian groups are proved in $8,9,10,11$. Before we move on to correspondences we pause to consider some elementary examples illustrating Theorem 1 .

2.1. Example. $H \cong C_{2} ; K \cong C_{2}$.

In Figure 3, $H=\{I, \sigma\}$ and $K=\{I, \rho\}$, where $\sigma$ is the involution which fixes the points marked by crosses, and $\rho$ is the involution which fixes the points marked by solid circles. The region exterior to the largest circle on the right is $D_{H}$, a fundamental domain for $H$, and the region exterior to the largest circle on the left is $D_{K}$, a fundamental domain for $K$. The intersection $\Delta=D_{H} \cap D_{K}$ is labelled ' 1 '. The other regions represented in the figure are $\sigma(\Delta)$ (labelled '2'), $\rho(\Delta)$ (labelled ' 3 '), $\sigma \rho(\Delta)$ (labelled ' 4 '), and $\rho \sigma(\Delta)$ (labelled ' 5 '). Only the first few 'layers' are shown. The central (grey) discs, $\sigma \rho \sigma\left(D_{H}\right)$ on the right hand side and $\rho \sigma \rho\left(D_{K}\right)$ on the left hand side, are in reality further subdivided into infinitely many annuli $(\sigma \rho \sigma(\Delta), \rho \sigma \rho(\Delta)$, and so on).

\subsection{Example. $H \cong C_{2} ; K \cong C_{3}$.}

In Figure $4, H=\{I, \sigma\}$ and $K=\left\{I, \rho, \rho^{2}\right\}$, where $\sigma$ is the involution which interchanges the right- and left-hand half-planes, fixing the origin and infinity, and $\rho$ is the rotation of order three which fixes the points marked with a solid circle. In the figure $D_{H}$ is the left-hand half-plane (bounded by the imaginary axis, marked $L$ ) and $D_{K}$ is the region exterior to the curve marked $M$. Their intersection $\Delta$ is labelled ' 1 ',$\sigma(\Delta)$ is labelled ' 2 '. The other regions represented in the figure are $\rho(\Delta)$ (labelled '3a'), $\rho^{2}(\Delta)$ (labelled '3b'), $\sigma \rho(\Delta)$ (labelled '4a'), $\sigma \rho^{2}(\Delta)$ (labelled ' $4 \mathrm{~b}$ '), $\rho \sigma(\Delta)$ (labelled ' $5 \mathrm{a}$ '), and $\rho^{2} \sigma(\Delta)$ (labelled ' $5 \mathrm{~b}$ '). Once again only the first few 'layers' are shown. The central (grey) discs $\sigma \rho \sigma\left(D_{H}\right)$ and $\sigma \rho^{2} \sigma\left(D_{H}\right)$ on the right hand side, and the central (grey) regions $\rho \sigma \rho\left(D_{K}\right) \cup \rho \sigma \rho^{2}\left(D_{K}\right)$ and $\rho^{2} \sigma \rho\left(D_{K}\right) \cup$ $\rho^{2} \sigma \rho^{2}\left(D_{K}\right)$ on the left hand side, are of course further subdivided into infinitely many further regions.

\section{A COMbination theOREM FOR COVERING CORRESPONDENCES}

In this section we present a generalisation of Theorem 1 in which the roles of the finite groups $H$ and $K$ of orders $|H|$ and $|K|$ are taken by the covering correspondences of rational maps $P$ and $Q$ of degrees $p$ and $q$, and the roles of fundamental 

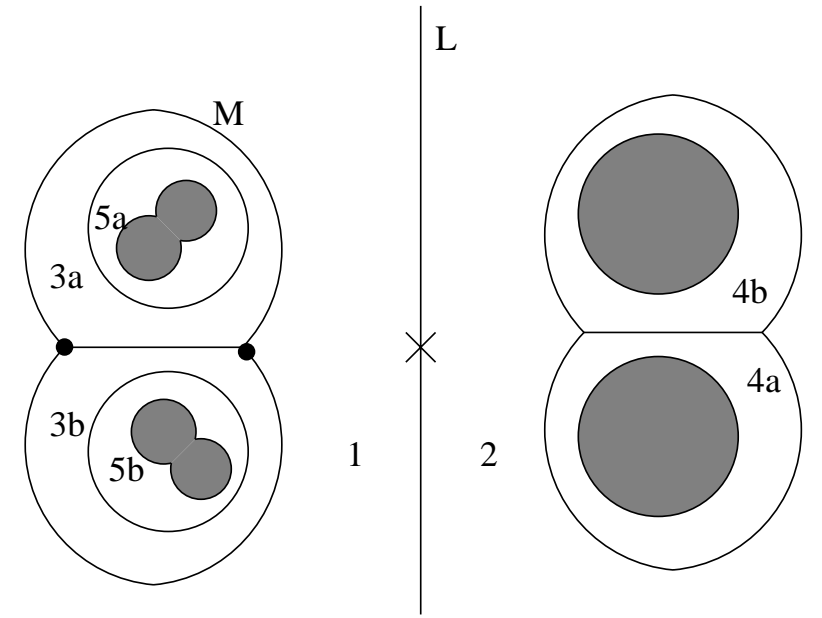

FIGURE 4.

domains for $H$ and $K$ are taken by transversals $D_{P}$ and $D_{Q}$ for $P$ and $Q$. The correspondences $\operatorname{Cov}^{P}, \operatorname{Cov}^{Q}, G a l^{P}$ and $G_{a l}^{Q}$ are multi-valued maps, a generic point having $p, q, p-1$ and $q-1$ images respectively. Each of these individual correspondences has all its grand orbits finite, but when we combine them by composition we obtain correspondences with (generically) infinite grand orbits. We shall be concerned with the dynamics of such combinations. Note that for us the dynamics (under composition) of the rational maps $P$ and $Q$ themselves is irrelevant.

We first establish some terminology. Let $f: z \rightarrow w$ be a holomorphic correspondence on the Riemann sphere, defined by a polynomial relation $p(z, w)=0$.

\section{Definitions.}

- The regular set $\Omega(f)$ of $f$ is the set of all points $z \in \hat{\mathbb{C}}$ which have a neighbourhood $U$ such that $U$ has only finitely many distinct returns under (mixed) iteration of $f$ and $f^{-1}$. Formally, we require [3] that there exist a positive integer $N$ such that

$$
\bigcup_{|e|<\infty} f^{e} \cap(U \times U) \subset \bigcup_{|e|<N} f^{e}
$$

(as graphs) where $e$ runs through all finite sequences $e_{1}, \ldots, e_{n}$ with each $e_{j}= \pm 1$, and where the length $n$ of $e$ is denoted $|e|$.

- The free regular set $\Omega^{\prime}(f)$ is the subset of $\Omega(f)$ consisting of points $z$ with the property that every point on the grand orbit of $z$ has a neighbourhood $U$ such that the only branch of (mixed) iteration of $f$ and $f^{-1}$ which returns some point of $U$ to $U$ is the identity, in other words

$$
\bigcup_{|e|<\infty} f^{e} \cap(U \times U)=I \cap(U \times U) .
$$

The free regular set is obtained from the regular set by puncturing it at the discrete set of points which have grand orbits containing singular points - that is to say points which have image under $f$ or $f^{-1}$ smaller in cardinality than the corresponding image of some neighbour. 
- The global limit set $\Lambda(f)$ is defined to be the complement in $\hat{\mathbb{C}}$ of $\Omega(f)$.

- A transversal $T$ for the action of $f$ on $\Omega=\Omega(f)$ is a maximal subset of $\Omega$ on which the grand orbit quotient map $\Omega \rightarrow \Omega / f$ is injective.

- A fundamental set $S$ for the action of $f$ on $\Omega^{\prime}=\Omega^{\prime}(f)$ is a transversal for the action of $f$ on $\Omega^{\prime}$.

In Example 1.2 the regular set $\Omega$ is the whole of $\hat{\mathbb{C}}$ and the free regular set $\Omega^{\prime}$ is $\hat{\mathbb{C}}-\{ \pm 1, \pm 2, \infty\}$. The set $D_{Q}$ described in this example is a transversal for the action of $\operatorname{Cov}^{Q}$ on $\Omega$. To make $D_{Q}$ into a fundamental set $D_{Q}^{\prime}$ for the action of $\operatorname{Cov}^{Q}$ on $\Omega^{\prime}$ we must remove the points $\infty,+1$ and +2 .

- If $P$ and $Q$ are rational maps we denote by $\operatorname{Cov}^{P} \circ \operatorname{Cov}^{Q}$ the composite of $\operatorname{Cov}^{Q}$ followed by $\operatorname{Cov}^{P}$ (generically a $(p q: p q)$ correspondence), and we denote by $\operatorname{Cov}^{P} * \operatorname{Cov}^{Q}$ the equivalence relation on $\hat{\mathbb{C}}$ generated by $\operatorname{Cov}^{P}$ and $\operatorname{Cov}^{Q}$.

- We call a sequence of points $z_{0}, \ldots, z_{n}$ in $U$ a reduced orbit linking $z_{0}$ to $z_{n}$ if alternate pairs $\left(z_{i}, z_{i+1}\right)$ lie in $\operatorname{Cov}^{P}$ and $\operatorname{Cov}^{Q}$ and no $z_{i+1}=z_{i}$.

Note that since $\operatorname{Cov}^{P} \circ \operatorname{Cov}^{P}=\operatorname{Cov}^{P}$ and $\operatorname{Cov}^{Q} \circ \operatorname{Cov}^{Q}=\operatorname{Cov}^{Q}$ a pair of points $(z, w) \in \hat{\mathbb{C}} \times \hat{\mathbb{C}}$ lies in $\operatorname{Cov}^{P} * \operatorname{Cov}^{Q}$ if and only if there exists a reduced orbit linking them. Observe also that the inverse of a reduced orbit is a reduced orbit, and that we can compose reduced orbits by concatenation followed by reduction.

- We say that $\operatorname{Cov}^{P} * \operatorname{Cov}^{Q}$ acts freely on a subset $U \subset \hat{\mathbb{C}}$ if $U$ is invariant under both $\operatorname{Cov}^{P}$ and $\operatorname{Cov}^{Q}$, each $z \in U$ has $p$ distinct images under $\operatorname{Cov}^{P}$ and $q$ distinct images under $\operatorname{Cov}^{Q}$ (where $p$ and $q$ are the degrees of $P$ and $Q$ ), and the only reduced orbit linking any point $z \in U$ to itself is the trivial one.

Theorem 2. Let $P$ and $Q$ be rational maps $\hat{\mathbb{C}} \rightarrow \hat{\mathbb{C}}$, let $D_{P}, D_{Q}$ be transversals for $P$ and $Q$ respectively having interiors $D_{P}^{\circ}, D_{Q}^{\circ}$ with $\overline{D_{P}^{\circ}}=\overline{D_{P}}$ and $\overline{D_{Q}^{\circ}}=\overline{D_{Q}}$, and suppose that $D_{P}^{\circ} \cup D_{Q}^{\circ}=\hat{\mathbb{C}}$. Let $D_{P}^{\prime}$ and $D_{Q}^{\prime}$ denote the sets obtained by removing from $D_{P}$ and $D_{Q}$ all points on grand orbits under $\operatorname{Cov}^{P} * \operatorname{Cov}^{Q}$ of critical points of $P$ and $Q$. Let $\Delta$ denote $D_{P} \cap D_{Q}$ and $\Delta^{\prime}$ denote $D_{P}^{\prime} \cap D_{Q}^{\prime}$. Then:

- $\mathcal{F}=\operatorname{Cov}^{P} * \operatorname{Cov}^{Q}$ acts freely on $\Omega\left(\mathcal{F}, \Delta^{\prime}\right)$, the union of grand orbits of points of $\Delta^{\prime}$ under $\mathcal{F}$, and $\Delta^{\prime}$ is a fundamental set for this action.

- $\Omega\left(\mathcal{F}, \Delta^{\prime}\right) \subset \Omega^{\prime}(\mathcal{F})$, the free regular set for the action of $\mathcal{F}$ on $\hat{\mathbb{C}}$.

- $\Delta$ is a transversal for the action of $\mathcal{F}$ on the union $\Omega(\mathcal{F}, \Delta)$ of grand orbits of points of $\Delta$ under $\mathcal{F}$.

- $\Omega(\mathcal{F}, \Delta) \subset \Omega(\mathcal{F})$, the regular set of $\mathcal{F}$.

- $\hat{\mathbb{C}}-\Omega(\mathcal{F}, \Delta)$ is the disjoint union of $\Lambda_{+}(\mathcal{G})=\bigcap_{0}^{\infty} \mathcal{G}^{n}\left(D_{Q}\right)$ and $\Lambda_{-}(\mathcal{G})=$ $\bigcap_{0}^{\infty} \mathcal{G}^{-n}\left(D_{P}\right)$, where $\mathcal{G}=G_{a l}^{P} \circ G a l^{Q}$ and $\mathcal{G}^{n}\left(D_{Q}\right)$ denotes the set of all images of points of $D_{Q}$ under the composition of $n$ iterates of the multi-valued map $\mathcal{G}$.

- if $\mathcal{G}\left(D_{Q}^{\circ}\right)$ and $\mathcal{G}^{-1}\left(D_{P}^{\circ}\right)$ are topological discs, then $\Lambda_{+}$and $\Lambda_{-}$are homeomorphic to the filled Julia sets $K\left(R_{+}\right)$and $K\left(R_{-}\right)$of polynomial maps $R_{+}$and $R_{-}$, each of degree $(p-1)(q-1)$. The restrictions of $\mathcal{G}^{-1}$ to domain and range $\Lambda_{+}$, and of $\mathcal{G}$ to domain and range $\Lambda_{-}$, are both single-valued maps and are conjugate to the maps $R_{+}$on $K\left(R_{+}\right)$and $R_{-}$on $K\left(R_{-}\right)$respectively, conformally on interiors.

Before proving the theorem we illustrate in Figure 5 some similarities to (and differences from) the group case. This figure is not intended to be a precise plot for an actual pair of rational functions $P$ and $Q$ but rather to represent the various regions schematically. In the figure, $P$ is a rational function of degree 4 , having a triple critical point $c_{0}$, a simple critical point $c_{1}$ and a double critical point $c_{2}$ (all marked by crosses). The region $D_{P}$ exterior to the closed curve $L$ is a transversal 

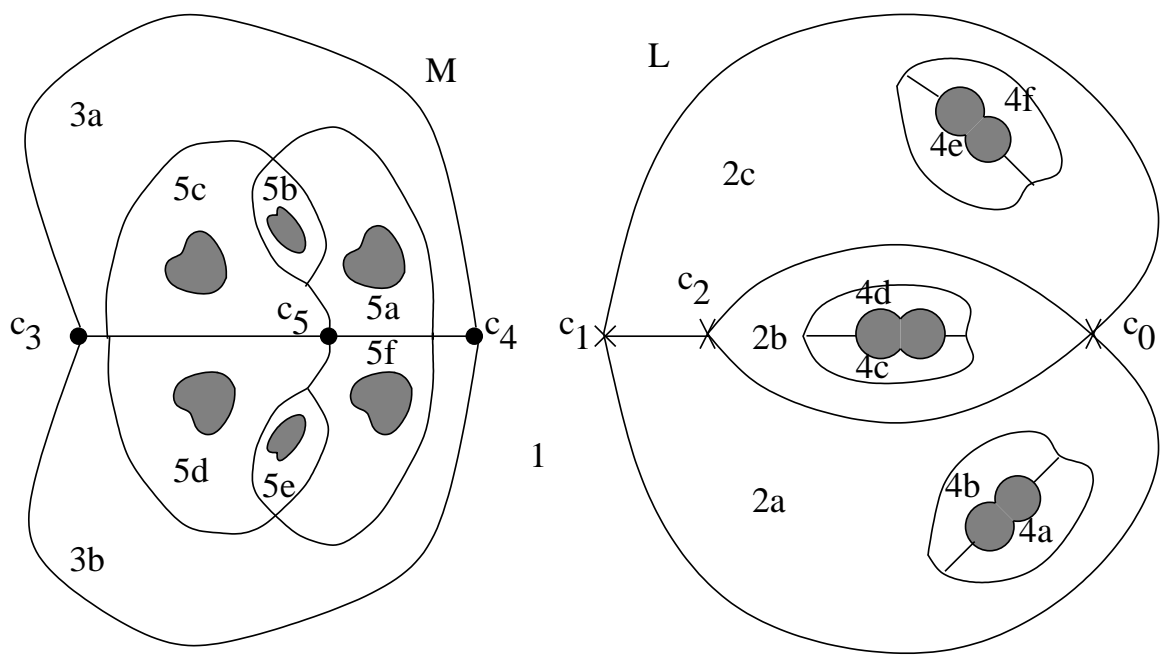

FIGURE 5 .

for $P$. The function $Q$ is of degree 3 , having one double critical point $c_{3}$ and two simple critical points $c_{4}$ and $c_{5}$ (all marked by solid circles). The region $D_{Q}$ exterior to the closed curve $M$ is a transversal for $Q$. The intersection $\Delta=D_{P} \cap D_{Q}$ is labelled ' 1 '. The set $\operatorname{Gal}^{P}(\Delta)$ is made up of three 'tiles' (copies of $\Delta$ ) labelled ' $2 \mathrm{a}$ ', '2b', and '2c' in the figure; $\mathrm{Gal}^{Q}(\Delta)$ is made up of the two 'tiles' labelled ' $3 \mathrm{a}$ ' and ' $3 \mathrm{~b}$ '; $\mathrm{Gal}^{P} \circ \mathrm{Gal}^{Q}(\Delta)$ is made up of the six tiles labelled '4a' to ' $4 \mathrm{f}$ '; and $G l^{Q} \circ G a l^{P}(\Delta)$ is made up of the six tiles labelled '5a' to ' $5 \mathrm{f}$ '. Only the first few 'layers' are shown. The six inner (grey) regions on the left (which together make up $\left.G a l^{Q} \circ G a l^{P} \circ G a l^{Q}\left(D_{Q}\right)\right)$ are in reality further subdivided, as are the six inner (grey) regions on the right (which together make up $G^{P} l^{P} \circ G a l^{Q} \circ G a l^{P}\left(D_{P}\right)$ ). The tiling is closely analogous to tilings we have seen in the group case, for example that in Figure 4. However there are differences: for example in Figure 5 the set $G_{a l}{ }^{Q} \circ \operatorname{Gal}^{P}\left(D_{P}\right)$ (which consists of the tiles numbered ' 5 ' together with the grey regions they surround) is a topological disc, double-covering the disc $\operatorname{Gal}^{P}\left(D_{P}\right)$ (the region interior to $L$ ) with ramification point $c_{5}$, whereas in Figure 4 the set $G l^{Q}$ 。 $\operatorname{Gal}^{P}\left(D_{P}\right)$ has two components, each single-covering $\operatorname{Gal}^{P}\left(D_{P}\right)$. If for sufficiently large $n$ all critical points are outside $\left(G a l^{Q} \circ G a l^{P}\right)^{n}\left(D_{P}\right) \cup\left(G a l^{P} \circ G a l^{Q}\right)^{n}\left(D_{Q}\right)$ (as they are in Figure 5) the limit set will be a Cantor set, but this will not always be the case, as we shall see in Section 4 .

We now proceed to the proof of Theorem 2 .

Proof. Since $D_{P}$ is a transversal to $P$, writing $D_{P}^{c}$ for $\hat{\mathbb{C}}-D_{P}$ we have

$$
\operatorname{Gal}^{P}\left(D_{P}\right)=D_{P}^{c} \cup\left(\operatorname{Gal}^{P}\left(D_{P}\right) \cap D_{P}\right) .
$$

But $D_{Q}=D_{P}^{c} \cup \Delta$ and $\operatorname{Gal}^{P}\left(D_{P}\right) \cap D_{P} \subset \partial D_{P} \subset D_{Q}$ so

$$
D_{Q}=\operatorname{Gal}^{P}\left(D_{P}\right) \cup \Delta .
$$

By inserting into this expression the analogous equality for $D_{P}$ we find that

$$
D_{Q}=\left(G a l^{P} \circ G a l^{Q}\right)\left(D_{Q}\right) \cup G a l^{P}(\Delta) \cup \Delta .
$$


Similarly

$$
D_{P}=\left(G a l^{Q} \circ G a l^{P}\right)\left(D_{P}\right) \cup G a l^{Q}(\Delta) \cup \Delta .
$$

Writing $\mathcal{G}$ for the composition $\mathrm{Gal}^{P} \circ \mathrm{Gal}^{Q}$, so $\mathcal{G}^{-1}=\mathrm{Gal}^{Q} \circ \mathrm{Gal}^{P}$, we set

$$
\begin{aligned}
& \mathcal{A}_{2 n}=\mathcal{G}^{n}(\Delta), \quad \mathcal{A}_{2 n+1}=\mathcal{G}^{n} \circ \operatorname{Gal}^{P}(\Delta), \\
& \mathcal{B}_{2 n}=\mathcal{G}^{-n}(\Delta), \quad \mathcal{B}_{2 n+1}=\mathcal{G}^{-n} \circ \operatorname{Gal}^{Q}(\Delta)
\end{aligned}
$$

$\left(\right.$ so $\left.\mathcal{A}_{0}=\mathcal{B}_{0}=\Delta\right)$. The expressions above for $D_{Q}$ and $D_{P}$ generalise inductively to

$$
D_{Q}=\mathcal{G}^{n}\left(D_{Q}\right) \cup \bigcup_{m=0}^{2 n-1} \mathcal{A}_{m}, \quad D_{P}=\mathcal{G}^{-n}\left(D_{P}\right) \cup \bigcup_{m=0}^{2 n-1} \mathcal{B}_{m}
$$

and (by definition) the union of grand orbits of points of $\Delta$ is

$$
\Omega(\mathcal{F}, \Delta)=\Delta \cup \bigcup_{n \geq 1} \mathcal{A}_{n} \cup \bigcup_{n \geq 1} \mathcal{B}_{n}
$$

We remark that the restricted maps $G^{P}{ }^{P}: \mathcal{A}_{n+1} \rightarrow \mathcal{B}_{n}$ and $G a l^{Q}: \mathcal{B}_{n+1} \rightarrow \mathcal{A}_{n}$ are single-valued surjections for all $n \geq 0$, by Lemma $1(\mathrm{i})$, since $\mathcal{B}_{n} \subset D_{P}$ and $\mathcal{A}_{n} \subset D_{Q}$.

Clearly no $\overline{\mathcal{A}_{m}}$ with $m>0$ can meet any $\overline{\mathcal{B}_{n}}$ with $n>0$ as the complements $D_{P}^{c}$ of $D_{P}$ and $D_{Q}^{c}$ of $D_{Q}$ have disjoint closures. We shall need to ascertain how far there can be non-empty intersections amongst the $\overline{\mathcal{A}_{n}}$ or the $\overline{\mathcal{B}_{n}}$. Observe that

$$
\operatorname{Gal}^{P}\left(\overline{D_{Q}^{c}}\right) \cap \overline{D_{P}}=\emptyset
$$

by Lemma 1(iii), since $\overline{D_{Q}^{c}} \subset D_{P}^{\circ}$. From this we deduce firstly that

$$
\operatorname{Gal}^{P}\left(\overline{\left.\operatorname{Gal}^{Q}\left(D_{Q}\right)\right)} \subset\left(D_{P}^{c}\right)^{\circ}\right.
$$

and so

$$
\overline{\mathcal{G}\left(D_{Q}\right)} \subset \overline{D_{P}^{c}} \subset D_{Q}^{\circ}
$$

which we shall need later, when we apply the 'Straightening Theorem' of Douady and Hubbard. We deduce secondly that for any $n \geq 2$ the set $\overline{\mathcal{A}_{0}}=\bar{\Delta}$ is disjoint from $\overline{\mathcal{A}_{n}}$, since the former is contained in $\overline{D_{P}}$ and the latter in $\operatorname{Gal}^{P}\left(\overline{D_{Q}^{c}}\right)$ (note that $\mathrm{Gal}^{P}\left(\overline{D_{Q}^{c}}\right)$ is closed, being the inverse image of $\overline{D_{Q}^{c}}$ under a continuous map, since $\overline{D_{Q}^{c}} \subset D_{P}^{\circ}$ ). Similarly $\overline{\mathcal{B}_{0}} \cap \overline{\mathcal{B}_{n}}=\emptyset$ for any $n \geq 2$. Next, since $\overline{D_{P}^{c}} \subset D_{Q}^{\circ}$, we have $\operatorname{Gal}^{Q}\left(\overline{D_{P}^{c}}\right) \subset G a l^{Q}\left(D_{Q}^{\circ}\right)$ and thus, for all $n \geq 2, \overline{\mathcal{B}_{n}} \subset G a l^{Q}\left(D_{Q}^{\circ}\right)$ and so

$$
\overline{\mathcal{B}_{n}} \cap \overline{\mathcal{B}_{1}}=\overline{\mathcal{B}_{n}} \cap \overline{\mathcal{B}_{1}} \cap \operatorname{Gal}^{Q}\left(D_{Q}^{\circ}\right) .
$$

But for any $n \geq 3$ this set is empty since there is a map (a single-valued restriction of $G a l^{Q}$ ) from it into $\overline{\mathcal{A}_{n-1}} \cap \overline{\mathcal{A}_{0}}$, which we have just shown to be empty. Similarly $\overline{\mathcal{A}_{n}} \cap \overline{\mathcal{A}_{1}}=\emptyset$ for any $n \geq 3$. It now follows by induction that for any $m, n$ with $m-n \geq 2$ the intersections $\overline{\mathcal{A}_{m}} \cap \overline{\mathcal{A}_{n}}$ and $\overline{\mathcal{B}_{m}} \cap \overline{\mathcal{B}_{n}}$ are empty.

Our next objective is to show that each point $z \in \Omega(\mathcal{F}, \Delta)$ is linked to a unique point of $\Delta$ by a unique reduced orbit. By definition $z$ is mapped to a point of $\Delta$ by some sequence of iterates of $G_{a l}^{P}$ and $G a l^{Q}$, and hence linked to that point by a reduced orbit. Our task is to prove uniqueness. Suppose firstly that $z$ lies in just one $\mathcal{A}_{m}$ or $\mathcal{B}_{m}$, say $z \in \mathcal{A}_{2 n}=\mathcal{G}^{n}(\Delta)$ for definiteness. Then any two reduced orbits from points $z_{0}, z_{0}^{\prime} \in \Delta$ to $z$ must have successive points in $\Delta=$ $\mathcal{A}_{0}, \mathcal{B}_{1}, \mathcal{A}_{2}, \mathcal{B}_{3}, \ldots, \mathcal{B}_{2 n-1}, \mathcal{A}_{2 n}$. But consider the reverse orbits: since the maps 
from $\mathcal{A}_{m}$ to $\mathcal{B}_{m-1}$ and $\mathcal{B}_{m}$ to $\mathcal{A}_{m-1}$ defined by $G a l^{P}$ and $G a l^{Q}$ are single-valued, the two reverse orbits are identical. Hence $z$ is indeed linked to a unique point $z_{0} \in \Delta$, by a unique reduced orbit. A similar argument applies to any $z$ which is in just one $\mathcal{A}_{2 n+1}, \mathcal{B}_{2 n}$ or $\mathcal{B}_{2 n+1}$. Next we turn our attention to the only other possibility for $z$, that it lies in two successive $\mathcal{A}_{n}$ or two successive $\mathcal{B}_{n}$. First observe that $\mathcal{A}_{0}=\Delta$ can only meet $\mathcal{A}_{1}=\operatorname{Gal}^{P}(\Delta)$ at a fixed point of $\operatorname{Gal}^{P}$ in $D_{P}$ (necessarily on the boundary). The map $G a l^{Q}$ restricted to $\mathcal{B}_{1} \cup \mathcal{B}_{2} \rightarrow \mathcal{A}_{0} \cup \mathcal{A}_{1}$ sends any point in $\mathcal{B}_{1} \cap \mathcal{B}_{2}$ to a point in $\mathcal{A}_{0} \cap \mathcal{A}_{1}$, that is to say a fixed point of $\mathrm{Gal}^{P}$. Thus the maps $\mathrm{Gal}^{Q}: \mathcal{B}_{1} \rightarrow \Delta$ and $\mathrm{Gal}^{P} \circ \mathrm{Gal}^{Q}: \mathcal{B}_{2} \rightarrow \Delta$ agree on $\mathcal{B}_{1} \cap \mathcal{B}_{2}$. Similarly Gal ${ }^{Q} \circ G a l^{P}: \mathcal{A}_{2} \cup \mathcal{A}_{3} \rightarrow \mathcal{A}_{0} \cup \mathcal{A}_{1}$ sends any point of $\mathcal{A}_{2} \cap \mathcal{A}_{3}$ to a fixed point of $G_{a l}^{P}$ - in other words the maps $G_{a l}{ }^{Q} \circ G^{P} l^{P}: \mathcal{A}_{2} \rightarrow \Delta$ and $\mathrm{Gal}^{P} \circ \mathrm{Gal}^{Q} \circ \mathrm{Gal}^{P}: \mathcal{A}_{3} \rightarrow \Delta$ agree on $\mathcal{A}_{2} \cap \mathcal{A}_{3}$. A similar argument applies to any point in the intersection of any successive pair of $\mathcal{A}_{n}$ 's or $\mathcal{B}_{n}$ 's. Thus each point of $\Omega(\mathcal{F}, \Delta)$ is indeed on the grand orbit under $\mathcal{F}$ of a unique point of $\Delta$ and is linked to that point by a unique reduced orbit. Moreover it also follows that between any two points $z$ and $w$ on the grand orbit of $z_{0} \in \Delta$ there is a unique reduced orbit, for if there were two such orbits, concatenation with the reduced orbit from $z_{0}$ to $z$ (followed if necessary by reduction) would give two different reduced orbits from $z_{0}$ to $w$.

Now consider the set $\Omega\left(\mathcal{F}, \Delta^{\prime}\right)$, where all grand orbits of critical points have been removed. Each $z \in \Omega\left(\mathcal{F}, \Delta^{\prime}\right)$ has exactly $p$ images under $\operatorname{Cov}^{P}$ and $q$ images under $\operatorname{Cov}^{Q}$, and together with our observation concerning unique reduced orbits this completes the proof that $\operatorname{Cov}^{P} * \operatorname{Cov}^{Q}$ acts freely on $\Omega\left(\mathcal{F}, \Delta^{\prime}\right)$. As each grand orbit contains exactly one point of $\Delta^{\prime}$, the latter is a fundamental set for the action. Moreover since every point $z$ of $\Delta^{\prime}$ is interior to either $\Delta^{\prime} \cup \operatorname{Gal}^{P}\left(\Delta^{\prime}\right)$ or to $\Delta^{\prime} \cup G a l^{Q}\left(\Delta^{\prime}\right)$, and the projections from $\Omega\left(\mathcal{F}, \Delta^{\prime}\right)$ to these sets are regular covers (being complex analytic, and having no critical points), $\Omega\left(\mathcal{F}, \Delta^{\prime}\right)$ is contained in $\Omega^{\prime}(\mathcal{F})$, the free regular set for the action of $\mathcal{F}$ on $\hat{\mathbb{C}}$.

Returning to the general situation where critical orbits are present, and using our single-valued projections from $\Omega(\mathcal{F}, \Delta)$ onto $\Delta$, we can subdivide each $\mathcal{A}_{n}$ and $\mathcal{B}_{n}$ into finitely many 'tiles' (copies of $\Delta$, possibly intersecting at boundaries). See Figure 5 for a schematic example. Note that we may have to make choices of cuts between critical points in order to define some parts of the boundaries (as in Example 1.2, Figure 2). Since each $\overline{\mathcal{A}_{n}}$ and $\overline{\mathcal{B}_{n}}$ can only meet two others, the tiling is locally finite and the grand orbits of critical points are discrete. Hence for any $z \in \Omega(\mathcal{F}, \Delta)$ on a critical grand orbit there exists a neighbourhood $U$ of $z$ such that any branch of $\mathcal{F}$ which returns some point of $U$ to $U$ fixes $z$, and there are only finitely many such branches. For points $z$ not on critical grand orbits we already know that there is only one such branch, the identity. Thus $\Omega(\mathcal{F}, \Delta) \subset \Omega(\mathcal{F})$, the regular set of $\mathcal{F}$. We have already established that each point of $\Omega(\mathcal{F}, \Delta)$ has a unique point of $\Delta$ in its grand orbit, that is to say $\Delta$ is a transversal to the action.

It remains to prove the statements concerning the structure of $\Lambda_{+}$and $\Lambda_{-}$. Observe firstly that

$$
\mathcal{G}^{-1}: \mathcal{G}\left(D_{Q}^{\circ}\right) \rightarrow D_{Q}^{\circ}
$$

is a (single-valued) complex analytic map of degree $(p-1)(q-1)$, being the composite of restrictions of $G_{a l}^{P}$ and $G a l^{Q}$ which are (single-valued) complex analytic by Lemma 1(ii). Moreover this restriction of $\mathcal{G}^{-1}$ has the property that the closure of its domain is contained in the interior of its image, from our observation earlier 
in the proof that

$$
\overline{\mathcal{G}\left(D_{Q}\right)} \subset D_{Q}^{\circ}
$$

Thus if $\mathcal{G}\left(D_{Q}^{\circ}\right)$ is a topological disc, then our restriction of $\mathcal{G}^{-1}$ is a polynomiallike mapping in the sense of Douady and Hubbard [6] and we may deduce from their Straightening Theorem that on a neighbourhood of $\Lambda_{-}$the map $\mathcal{G}^{-1}$ is hybrid equivalent to a polynomial map $R_{-}$of degree $(p-1)(q-1)$. In particular $\Lambda_{-}$ is homeomorphic to the filled Julia set $K\left(R_{-}\right)$of $R_{-}$and $\mathcal{G}^{-1}$ restricted to $\Lambda_{-}$ is conjugate to $R_{-}$restricted to $K\left(R_{-}\right)$, conformally on the interior. The same reasoning proves the analogous results for $\Lambda_{+}$.

\section{Comments on Theorem 2.}

1. Although there is a unique reduced orbit linking any two points $z$ and $w$ on any grand orbit in $\Omega(\mathcal{F}, \Delta)$, it is only if the grand orbit is non-critical that this implies that the only branch of $\mathcal{F}$ fixing $z$ is the identity. Any critical point $c$ of $P$ is fixed by a branch of $\mathrm{Gal}^{P}$ as well as by the identity, and any point $z$ on the grand orbit of $c$ is fixed by first applying a sequence of iterations which carries $z$ to $c$, then applying a branch of $\mathrm{Gal}^{P}$ which fixes $c$, and finally applying the reverse sequence of iterations to carry $c$ back to $z$. The finite set of branches of $\mathcal{F}$ fixing a point $z$ on a critical grand orbit, described in the proof above, consists of concatenations of such sequences.

2. The orbit space $\Omega(\mathcal{F}, \Delta) / \mathcal{F}$ is an orbifold, with a finite set of cone points (corresponding to grand orbits of $\mathcal{F}$ containing singular points). Deleting the cone points one obtains the punctured Riemann surface $\Omega\left(\mathcal{F}, \Delta^{\prime}\right) / \mathcal{F}([3])$.

3. In contrast to the group case the inclusions $\Omega\left(\mathcal{F}, \Delta^{\prime}\right) \subset \Omega^{\prime}(\mathcal{F})$ and $\Omega(\mathcal{F}, \Delta) \subset$ $\Omega(\mathcal{F})$ need not be equalities. Equivalently the inclusion $\Lambda(\mathcal{F}) \subset \Lambda_{+}(\mathcal{G}) \cup \Lambda_{-}(\mathcal{G})$ need not be an equality (see the examples and comment in section 4.1).

4. If $p=2$ and $G a l^{Q}\left(D_{Q}^{\circ}\right)$ is a disc, then $\mathcal{G}\left(D_{Q}^{\circ}\right)$ is a disc, since $G a l^{P}$ is a bijection. 5. If $p=2$ the involution $\mathrm{Gal}^{P}$ conjugates $\mathcal{G}$ to $\mathcal{G}^{-1}$. In particular $\mathrm{Gal}^{P}$ maps $\Lambda_{-}$ homeomorphically onto $\Lambda_{+}$and conjugates the action of $\mathcal{G}$ on $\Lambda_{-}$to that of $\mathcal{G}^{-1}$ on $\Lambda_{+}$. For $p$ and $q$ both greater than 2, the best we can say is that $\operatorname{Gal}^{P}$ and $G_{a l}^{Q}$ define holomorphic maps $f_{0}: \Lambda_{-} \rightarrow \Lambda_{+}$and $f_{1}: \Lambda_{+} \rightarrow \Lambda_{-}$of degrees $p-1$ and $q-1$ respectively, and that on suitable neighbourhoods of $\Lambda_{-}$and $\Lambda_{+}$the composition $f_{1} \circ f_{0}$ is hybrid equivalent to a polynomial map $R_{-}$and $f_{0} \circ f_{1}$ is hybrid equivalent to a polynomial map $R_{+}$. It is not clear whether $R_{-}$and $R_{+}$themselves need be compositions in general, nor whether $\Lambda_{+}$need be homeomorphic to $\Lambda_{-}$.

6. If $\mathcal{G}^{-1}\left(D_{P}^{\circ}\right)$ is a disjoint union of discs (as it is in the group case), then $\mathcal{G}$ restricted to it is a generalised polynomial mapping in a suitable sense and one can draw appropriate conclusions about the structure of $\Lambda_{-}$. For example if $\mathcal{G}^{-1}\left(D_{P}^{\circ}\right)$ consists of $(p-1)(q-1)$ disjoint discs (as it does in the group case if $D_{P}^{\circ}$ is a disc) one can deduce that $\Lambda_{-}$is necessarily a Cantor set.

\section{Matings of Hecke groups with polynomial maps}

4.1. Mating the modular group $C_{2} * C_{3}$ with quadratic maps. Matings of quadratic maps with the modular group were first constructed (modulo a technical difficulty) in [1]. These are quadratic correspondences which on a topological disc contained in the Riemann sphere are conjugate to the modular group $P S L(2, \mathbb{Z})\left(\cong C_{2} * C_{3}\right)$, acting on complex upper half-space by $z \rightarrow z+1, z \rightarrow z /(z+1)$, and on the complement of this disc have branches conjugate to $q_{c}: z \rightarrow z^{2}+c$ 
and its inverse, acting on the filled Julia set $K\left(q_{c}\right)$ of $q_{c}$. Below we repeat the construction (modulo the same technical difficulty), making use of Theorem 2 to simplify the description, and we show how representations of $C_{2} * C_{3}$ with limit sets which are Cantor sets may also be mated with quadratic maps, now without any technical problem.

Let $P$ be a rational map of degree two, and $Q$ be a rational map of degree three with one double critical point and two simple ones. Without loss of generality we may take:

$$
\begin{gathered}
P(z)=z^{2} \\
Q(z)=\left(\frac{a z+1}{z+1}\right)^{3}-3 k\left(\frac{a z+1}{z+1}\right) .
\end{gathered}
$$

Then $P$ has critical points at 0 and $\infty$, and $\operatorname{Gal}^{P}(z)=-z$. The map $Q$ has a double critical point at $c_{0}=-1$ and single critical points at $c_{1}, c_{2}$, where

$$
c_{1}=\frac{\sqrt{k}-1}{a-\sqrt{k}} \text { and } c_{2}=\frac{-\sqrt{k}-1}{a+\sqrt{k}} .
$$

The correspondence $G a l^{Q}$ is given by

$$
z \rightarrow w \text { where }\left(\frac{a z+1}{z+1}\right)^{2}+\left(\frac{a z+1}{z+1}\right)\left(\frac{a w+1}{w+1}\right)+\left(\frac{a w+1}{w+1}\right)^{2}=3 k
$$

and the composite $\mathcal{G}=\mathrm{Gal}^{P} \circ \mathrm{Gal}^{Q}$ is given by

$$
z \rightarrow w \text { where }\left(\frac{a z+1}{z+1}\right)^{2}+\left(\frac{a z+1}{z+1}\right)\left(\frac{a w-1}{w-1}\right)+\left(\frac{a w-1}{w-1}\right)^{2}=3 k
$$

which is also the polynomial relation considered in [1]. For an appropriate set of values of the parameters $a$ and $k$ we can find transversals $D_{P}$ and $D_{Q}$ for $P$ and $Q$ satisfying the hypotheses of Theorem 2. An example is illustrated in Figure 6. Here $a$ and $\sqrt{k}$ are real, and $D_{Q}$ is as drawn in Figure 2 (but with the coordinate changed by a Möbius transformation, so that the point $\infty$ of Figure 2 has become the point $c_{0}=-1$ of Figure 6 ). In this figure $D_{P}$ is the left-hand half-plane, bounded by the imaginary axis $L$ and $D_{Q}$ is the region exterior to the heart-shaped curve $M$. The critical point 0 of $P$ is marked with a cross (the other critical point of $P$ is $\infty$ ); the critical points of $Q$ are marked with solid circles.

By Theorem 2 the Riemann sphere is now partitioned into two completely invariant sets, $\Omega(\mathcal{F}, \Delta)$ and $\Lambda(\mathcal{G})=\Lambda_{+}(\mathcal{G}) \sqcup \Lambda_{-}(\mathcal{G})$. If the values of $a$ and $k$ are such that the critical point $c_{2}$ is inside $\mathcal{G}^{-n}\left(D_{P}\right)$ for all $n \geq 0$, then $\Lambda_{-}(\mathcal{G})$ is connected and so is $\Lambda_{+}(\mathcal{G})=\operatorname{Gal}^{P}\left(\Lambda_{-}(\mathcal{G})\right)$, whence $\Omega(\mathcal{F}, \Delta)$ is a topological annulus. The situation is illustrated by Figure 7 . In this computer plot the red lines are the images of $L\left(=\partial D_{P}\right)$ (the imaginary axis) and the blue lines are the images of $M\left(=\partial D_{Q}\right)$. Both $\Lambda_{+}$and $\Lambda_{-}$(plotted in black) are homeomorphic to connected filled Julia sets of quadratic maps.

There are now two ways to obtain an action of $C_{2} * C_{3}$ : either we perturb the parameter $k$ until it takes the value 1 , in which case there is a conformal homeomorphism from $\Omega(\mathcal{F}, \Delta)$ to the upper half-plane $\mathcal{H}$, conjugating the action of the correspondence $\mathcal{F}$ on $\Omega$ to that of $\operatorname{PSL}(2, \mathbb{Z})\left(\cong C_{2} * C_{3}\right)$ on $\mathcal{H}$ (Method 1 below), or we keep the value of $k$ unchanged and identify an action of $C_{2} * C_{3}$ on a suitable modification of $\Omega(\mathcal{F}, \Delta)$, obtained by making cuts (Method 2 below). 


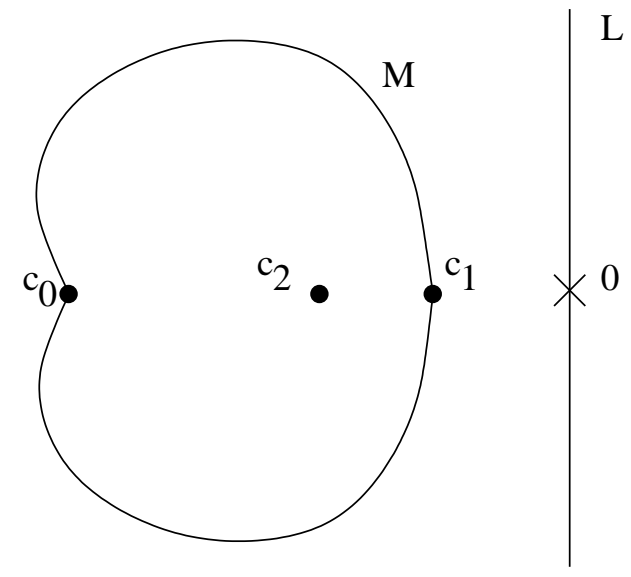

FIGURE 6.
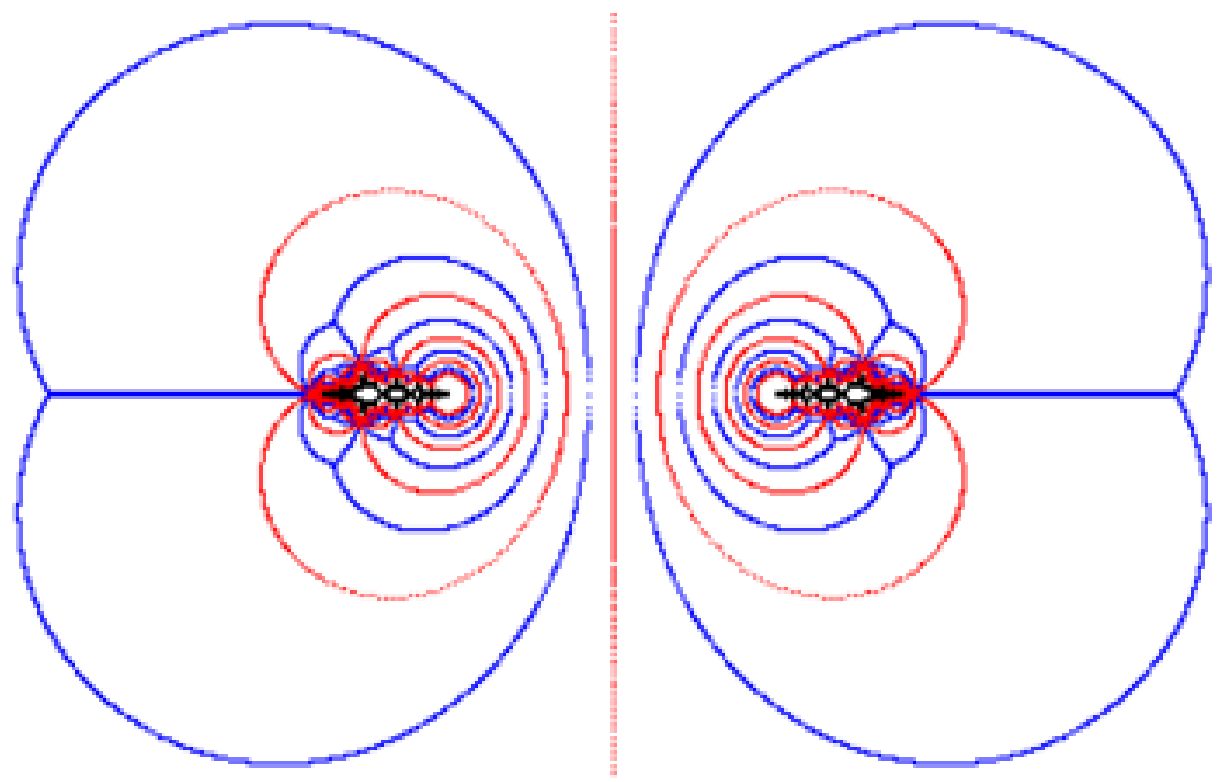

FigURE $7 . a=3.42 ; k=0.8$

Method 1 ([1]). Adjust the parameter $k$ to the value 1 . The critical point $c_{1}$ of $Q$ now moves to the origin which, we recall, is a critical point of $P$. Since $0 \in \partial D_{P}$ and $c_{1} \in \partial D_{Q}$ it is no longer possible for the interiors of $D_{P}$ and $D_{Q}$ to together cover $\hat{\mathbb{C}}$ but for values of the parameter $a$ in an appropriate set we can arrange that $D_{P}^{\circ} \cup D_{Q}^{\circ}=\hat{\mathbb{C}}-\{0\}$ [1. As $c_{1}$ has become 0 , the sets $\Lambda_{+}$and $\Lambda_{-}$now touch at the origin, and $\Omega(\mathcal{F}, \Delta)$ has become a (topological) disc. This disc contains just one critical point of $P$ (at $z=\infty)$, and just one critical point of $Q$ (at $z=-1$ ). As these critical points have multiplicities $p-1=1$ and $q-1=2$ respectively it follows that $\operatorname{Cov}^{P}$ and $\operatorname{Cov}^{Q}$ act as cyclic groups $C_{2}$ and $C_{3}$ on $\Omega(\mathcal{F}, \Delta)$ and 


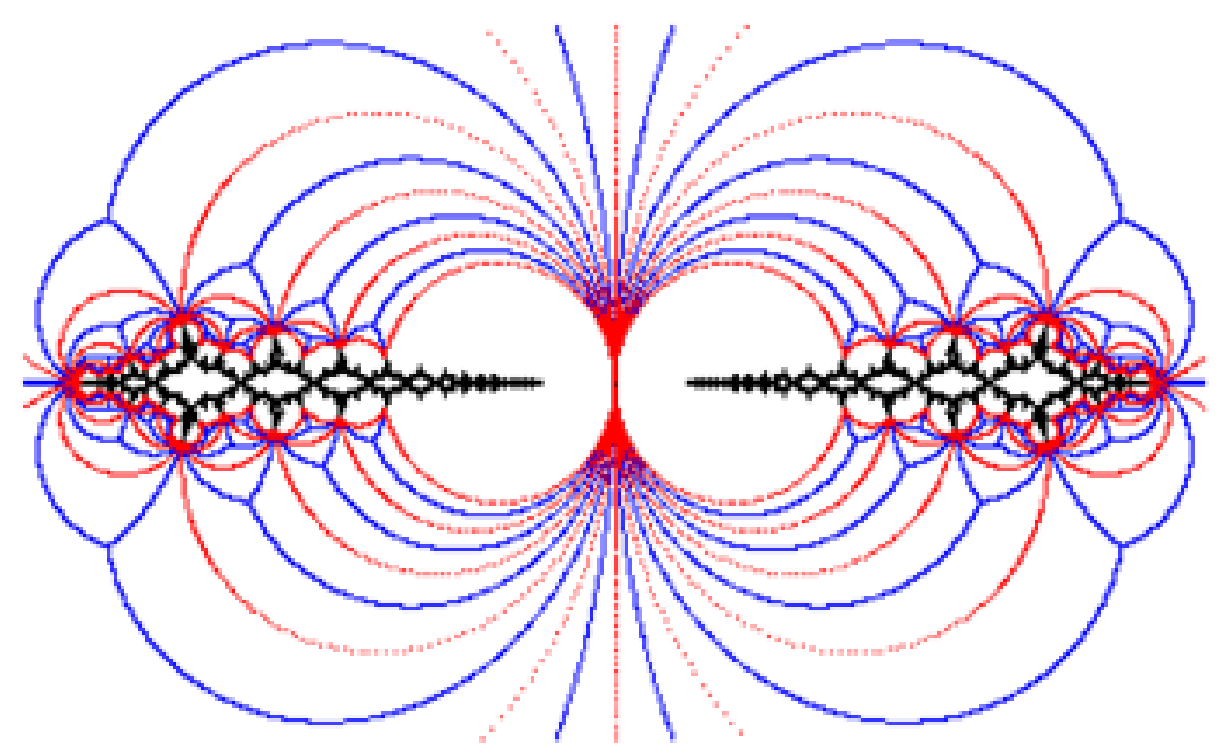

FiguRE 8. $a=4.3 ; k=1$

that we have a conformal bijection from $\Omega(\mathcal{F}, \Delta)$ to the complex upper half-plane conjugating the action of $\mathcal{F}$ to that of $\operatorname{PSL}(2, \mathbb{Z})$. An example with $k=1$ is plotted in Figure 8. Here we have zoomed in to twice the scale of Figure 7. See [1] for more computer plots of examples of matings obtained by setting $k=1$.

There are two drawbacks to this method. Firstly, by setting $k$ to be 1 we have violated the conditions of Theorem 2 in that the interiors of $D_{P}$ and $D_{Q}$ together only cover the punctured sphere $\hat{\mathbb{C}}-\{0\}$. While this causes no difficulty with the proof that the action of the correspondence $\mathcal{F}$ on $\Omega(\mathcal{F}, \Delta)$ is conjugate to that of $\operatorname{PSL}(2, \mathbb{Z})$ on the upper half-plane, it means that $\mathcal{G}: \mathcal{G}^{-1}\left(D_{P}^{\circ}\right) \rightarrow D_{P}^{\circ}$ is no longer strictly polynomial-like in the Douady-Hubbard sense: although $\mathcal{G}^{-1}\left(D_{P}^{\circ}\right) \subset D_{P}^{\circ}$, the closure of the first set is not contained in the second since the origin is on the boundary of both. This is the technical difficulty alluded to earlier: it remains a conjecture in the case $k=1$ that $\Lambda_{+}$and $\Lambda_{-}$are homeomorphic to the filled Julia set $K\left(q_{c}\right)$ of a quadratic map $q_{c}$ and that the appropriate restrictions of $\mathcal{G}$ and $\mathcal{G}^{-1}$ are topologically conjugate to the action of $q_{c}$ on $K_{c}$. The second drawback is that we have to adjust the value of $k$ to become 1 and we only obtain the standard action of $C_{2} * C_{3}$ (as $P S L(2, \mathbb{Z})$ on the upper half-plane). However we shall now see that there is an action of $C_{2} * C_{3}$ implicit within the correspondence without any alteration to $k$, and that this action is that of a faithful discrete representation of $C_{2} * C_{3}$ on $\hat{\mathbb{C}}$ having a Cantor set as its limit set.

Method 2. We assume that the conditions of Theorem 2 are satisfied, so that $D_{P}^{\circ} \cup D_{Q}^{\circ}=\hat{\mathbb{C}}$, and we assume also that $\Lambda_{+}$and $\Lambda_{-}$are each connected. The set $\Omega=\Omega(\mathcal{F}, \Delta)$ is now an annulus. Consider any curve $l$ which crosses $\Delta$ from the critical point 0 of $P$ to the critical point $c_{1}$ of $Q$, and let $\mathcal{C}$ denote the union of the grand orbits of points of $l$ under $\mathcal{F}$. The set $\mathcal{C}$ is illustrated in green in 
Figures 9 and 10. It consists of an arc joining $\Lambda_{+}$to $\Lambda_{-}$together with a countable union of line segments linking the points $\mathcal{G}^{n}\left(c_{1}\right), n \geq 1$ to $\Lambda_{+}$and linking the points $\mathcal{G}^{-n}\left(c_{1}^{\prime}\right), n \geq 0$ to $\Lambda_{-}$, where $c_{1}^{\prime}$ denotes the co-critical point of $Q$ corresponding to the critical point $c_{1}$. Cutting $\Omega$ along $\mathcal{C}$ yields a (topological) disc $\Omega^{\text {cut }}$. We claim there is a unique (up to Möbius conjugacy) faithful discrete representation $r$ of $C_{2} * C_{3}$ in $P S L(2, \mathbb{C})$, with connected regular set $\Omega_{r} \subset \hat{\mathbb{C}}$, such that there is an invariant (topological) disc $\mathcal{D} \subset \Omega_{r}$ and a conformal bijection $\Omega^{\text {cut }} \rightarrow \mathcal{D}$ conjugating $\mathcal{F}$ on $\Omega^{\text {cut }}$ to $C_{2} * C_{3}$ on $\mathcal{D}$. Thus we may regard $\mathcal{F}$ as a mating of this representation with the relevant quadratic map $q_{c}$.

To see why such a representation $r$ should exist we first consider the Fuchsian case ( $a$ and $k$ real), illustrated by Figure 9 . Here we take $l$ to be the segment of the real axis from the critical point 0 of $P$ to the critical point $c_{1}$ of $Q$. When we cut along $l$ and its images, $\Omega$ becomes a disc $\Omega^{\text {cut }}$ conformally homeomorphic to the upper half-plane via a bijection which carries the action of $\mathcal{F}$ to a (Fuchsian) action of $C_{2} * C_{3}$, that is to say a faithful discrete action with limit set (a Cantor set) contained in the boundary $\hat{\mathbb{R}}$ of the upper half-plane. This is self-evident: opening up the green cuts by halving angles at their end points is precisely what is needed to counteract the effect of the critical point $c_{1}$ and resolve the action of $\operatorname{Cov}^{Q}$ on the boundary of $\Omega^{\text {cut }}$ into that of a cyclic group $C_{3}$ of conformal bijections. As $P$ and $Q$ each have a single critical point in $\Omega^{\text {cut }}$ (of multiplicity $p-1$ and $q-1$ respectively), $\operatorname{Cov}^{P}$ and $\operatorname{Cov}^{Q}$ act on $\Omega^{c u t}$ as cyclic groups of conformal bijections conjugate to iterates of elliptic transformations of the upper half-plane.

Now to generalise the situation to the non-Fuchsian case (illustrated in Figure 10 ), we join the origin to $c_{1}$ (which is no longer necessarily real) by any curve $l$ in $\Delta$ which descends to a simple curve in the quotient orbifold $\Omega / \mathcal{F}$. When we cut along $l$ and its images under $\mathcal{F}$, the actions of $\operatorname{Cov}^{P}$ and $\operatorname{Cov}^{Q}$ on $\Omega$ become actions of cyclic groups of conformal bijections on $\Omega^{\text {cut }}$ just as before. Every representation $r$ of $C_{2} * C_{3}$ in $P S L(2, \mathbb{C})$ is equipped with an involution $\chi$ conjugating the generators of $C_{2}$ and $C_{3}$ to their inverses (on the Poincaré ball $\chi$ is rotation through $\pi$ about the common orthogonal to the fixed axes of $C_{2}$ and $C_{3}$ ). The orbifold $\Omega / \mathcal{F}$ is conformally isomorphic to $\Omega_{r} /\left\langle C_{2} * C_{3}, \chi\right\rangle$ for a unique $r$ (up to conjugacy), since by varying $r$ we run through the whole moduli space of complex structures on the topological type of this orbifold. Cutting $\Omega$ open along $\mathcal{C}$ to give $\Omega^{\text {cut }}$, and cutting $\Omega_{r}$ along the corresponding curves to yield two discs $\mathcal{D}$ and $\chi(\mathcal{D})$, we obtain the desired conjugacy from the action of $\mathcal{F}$ on $\Omega^{\text {cut }}$ to that of $C_{2} * C_{3}$ on $\mathcal{D}$. It can be shown by techniques of quasiconformal surgery that within the family of correspondences we are considering are matings of every quadratic map having a connected filled Julia set with every discrete faithful representation of $C_{2} * C_{3}$ having a connected regular set [5].

\section{Comments.}

1. As dynamically they partition the sphere into three disjoint regions $\Lambda_{-} \cong K\left(R_{-}\right)$, $\Lambda_{+} \cong K\left(R_{+}\right)$, and $\Omega(\mathcal{F}, \Delta) \cong \Omega_{r}$ one might also regard the correspondences above as matings of $R_{-}$(on $K\left(R_{-}\right)$) with $R_{+}$(on $K\left(R_{+}\right)$) along an action of $C_{2} * C_{3}$ (on $\left.\Omega_{r}\right)$. This is a legitimate viewpoint, but it must be remembered that $R_{-}$and $R_{+}$ are by no means independent. The sets $\Lambda_{-}$and $\Lambda_{+}$are not completely invariant, but only backwards invariant and forwards invariant respectively. Indeed, since $P$ has degree two in the construction considered above, $\left.R_{-} \mid K_{(} R_{-}\right)$is conjugate to $R_{+} \mid K\left(R_{+}\right)$(see Comment 5 at the end of Section 3). 
SHAUN BULLETT
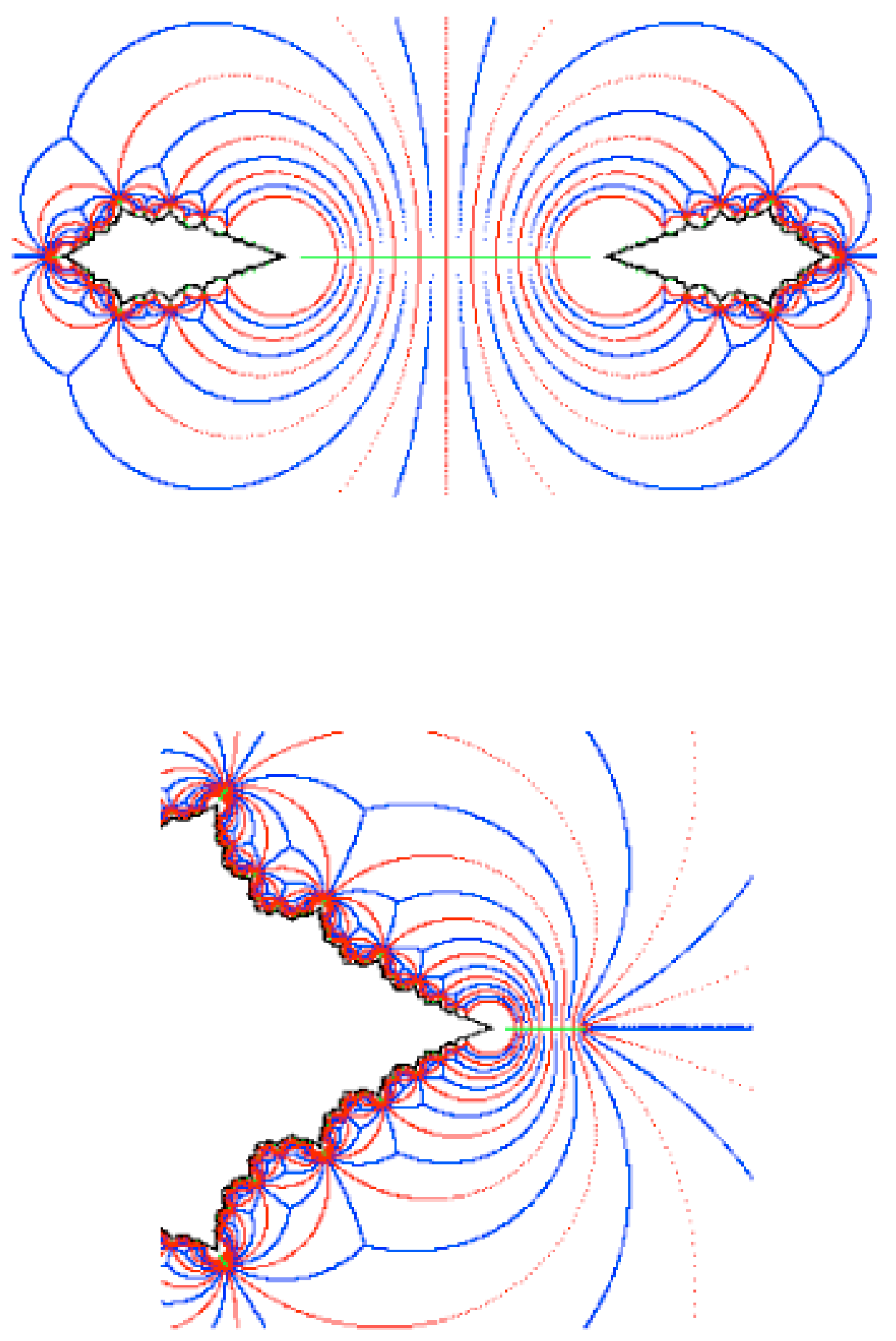

Figure $9 . a=4.1 ; k=0.9$ (and zoom) 

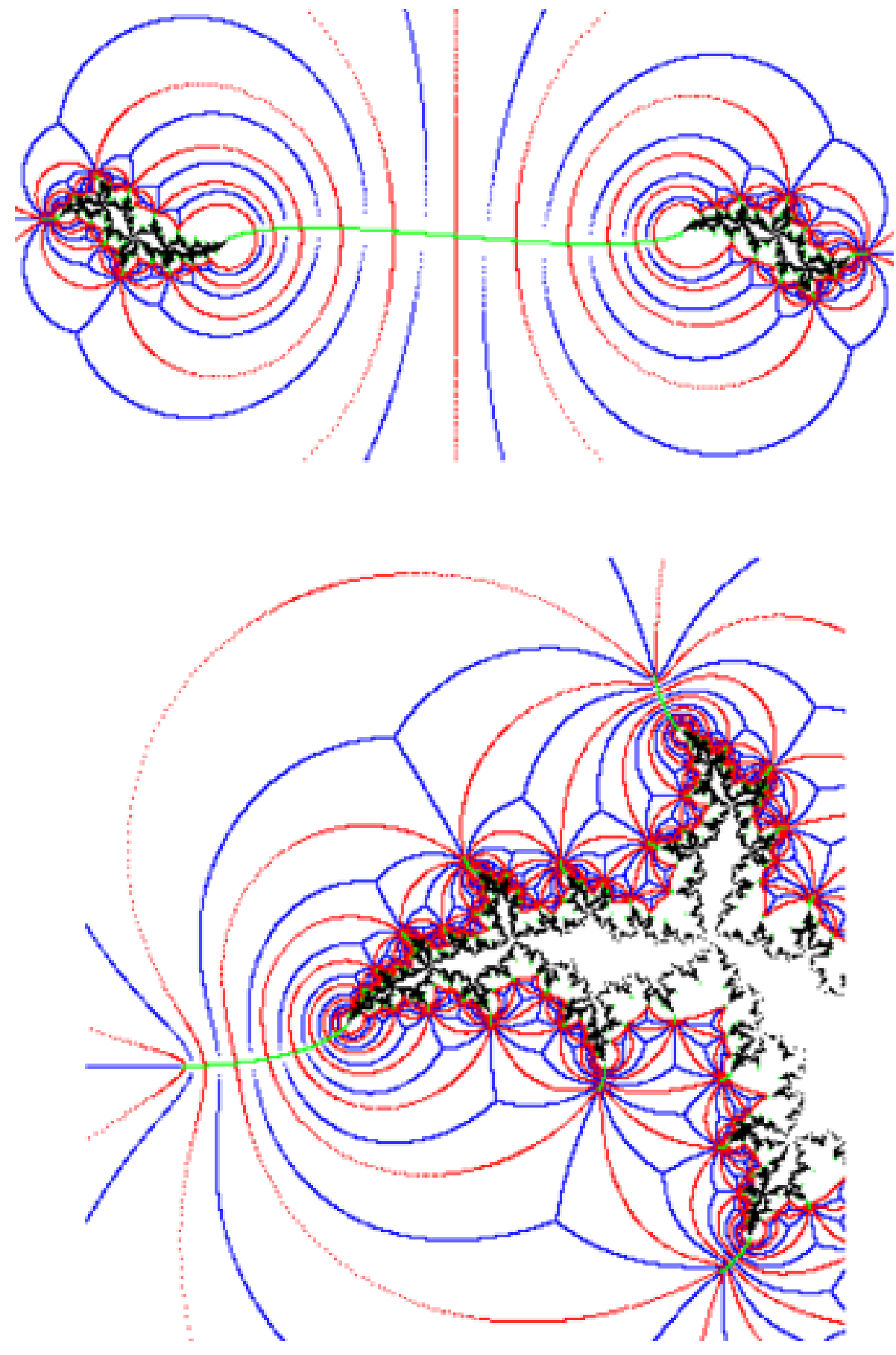

FigURE 10. $a=3.55+0.22 i ; k=0.8$ (and zoom) 


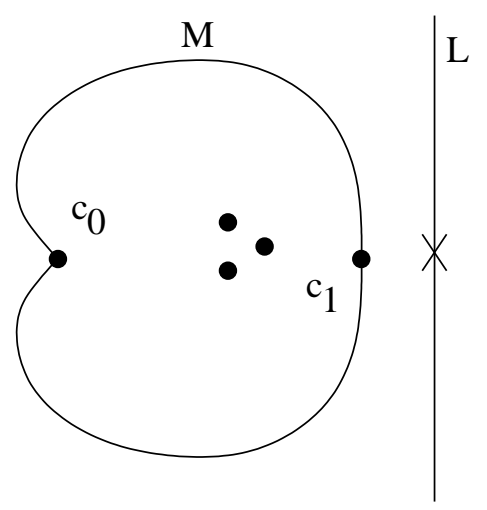

Figure 11.

2. It seems inevitable that some sort of construction involving cuts is needed for any tractable description of the matings of Method 2. There is no single involution on the regular set $\Omega_{r}$ of the representation of $C_{2} * C_{3}$ having as its quotient a space homeomorphic to $\Omega=\Omega(\mathcal{F}, \Delta)$ : in the description above we cut $\Omega_{r}$ along infinitely many curve segments (which join together the components of the Cantor set $\left.\mathbb{C}-\Omega_{r}\right)$ to divide it into two discs, $\mathcal{D}$ and $\chi \mathcal{D}$, and then obtain a copy of $\Omega$ by applying to one of these discs a boundary identification which uses a different conjugate of $\chi$ on each segment. To obtain a 'cut-free' description would involve passing to the universal cover of $\Omega_{r}$ and then quotienting by a group which is not finitely generated.

3. The region $\Omega=\Omega(\mathcal{F}, \Delta)$ may be only a part of the regular set $\Omega(\mathcal{F})$ as defined in the previous section. When the map $q_{c}$ has an attracting (but not superattracting) periodic orbit, or a neutral periodic orbit of multiplier $e^{2 \pi i \alpha}$ with $\alpha$ rational, the basin of this orbit is contained in $\Omega(\mathcal{F})$. Thus in this case $\Omega(\mathcal{F})$ contains the interior of $\Lambda_{+} \cup \Lambda_{-}$as well as $\Omega(\mathcal{F}, \Delta)$.

4.2. Mating the group $C_{2} * C_{q}$ with polynomial maps of degree $q-1$. For $q>3$ we proceed in a similar way as for the case $q=3$. We need rational maps $P$ and $Q$ of degree 2 and $q$ respectively, with critical points and transversals as depicted in Figure 11. Here, as in Figure $6, D_{P}$ is the left-hand half-plane, bounded by the imaginary axis $L$ and $D_{Q}$ is the region exterior to the heart-shaped curve $M$. The function $P$ is taken to be $z \rightarrow z^{2}$, with critical points 0 and $\infty$. We require the function $Q$ to have a critical point $c_{0}$ of multiplicity $q-1$, we require the transversal $D_{Q}$ to be such that $c_{0}$ lies on its boundary, we require that $Q$ also has a simple critical point $c_{1}$ on the boundary of $D_{Q}$, and we require that all the other $q-2$ critical points of $Q$ (counted with multiplicity) lie in the interior of the 'heart' $\widehat{\mathbb{C}}-D_{Q}$.

Any coordinate change on the domain of $Q$ alters $\operatorname{Cov}^{Q}$ by a conjugacy and any coordinate change on the range preserves $\operatorname{Cov}^{Q}$. Since $c_{0}$ has multiplicity $q-1$ we may choose coordinates for the domain and range of $Q$ for which this map becomes polynomial. In order that $\Lambda_{-}(\mathcal{G})$ be connected we need all the critical points of $Q$ which lie in the 'heart' $\hat{\mathbb{C}}-D_{Q}$ to remain inside $\mathcal{G}^{-n}\left(D_{P}\right)$ for all $n$. The simplest maps $Q$ to consider are those with just 3 critical points, of multiplicity $q-1,1$ and 

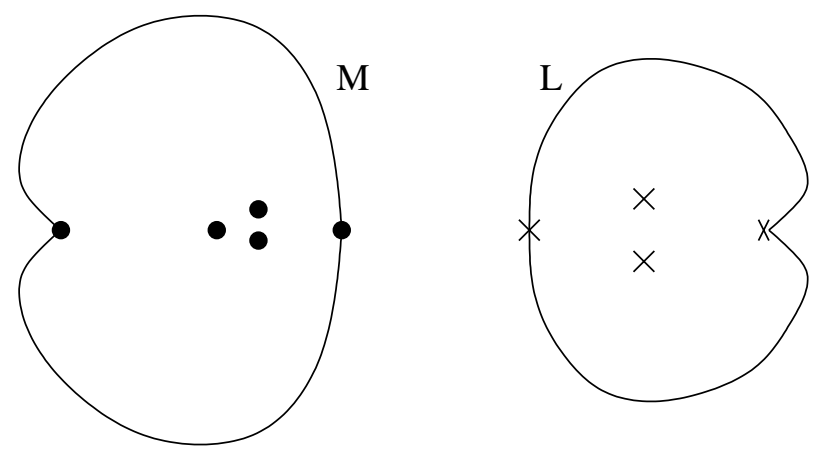

FIGURE 12.

$q-2$ respectively. These are the maps obtained by precomposing

$$
Q_{0}(z)=z\left(1+\frac{z}{q-1}\right)^{q-1}
$$

by a Möbius transformation, since $Q_{0}$ has a multiplicity $q-1$ critical point at $\infty$ (being a polynomial), a simple critical point at $z=(1-q) / q$ and a multiplicity $q-2$ critical point at $z=1-q$. Setting $P(z)=z^{2}$ and $Q(z)=Q_{0}(M z)$, where $M$ is the Möbius transformation sending $c_{0} \rightarrow \infty, c_{1} \rightarrow(1-q) / q$, and $c_{2} \rightarrow 1-q$, we obtain a two-parameter family of correspondences $\operatorname{Cov}^{P} * \operatorname{Cov}^{Q}$ with the same pattern of critical points as illustrated earlier in Figure 6, except that now $c_{2}$ is of multiplicity $q-2$. By the same methods as in the $q=3$ case, within this two-parameter family there are matings of $C_{q} * C_{2}$ with polynomials of the form $z \rightarrow z^{q-1}+c$ having connected filled Julia sets. (The particular form $z \rightarrow z^{q-1}+c$ follows from choosing $Q$ to have a multiplicity $q-2$ critical point: by choosing $Q$ to have different patterns of critical points we can mate $C_{q} * C_{2}$ with other types of polynomial of degree $q-1$.)

4.3. Mating the group $C_{p} * C_{q}$ with polynomial maps of degree $(p-1)(q-1)$. Now we need rational maps $P$ and $Q$ of degree $p$ and $q$ respectively, with critical points and transversals arranged as depicted in Figure 12. Here $D_{P}$ is the region outside the curve $L$ and $D_{Q}$ is that outside $M$. P has a multiplicity $p-1$ critical point on $L$, a single (simple) critical point elsewhere on $L$, and its other critical points lie in the interior of the 'heart' $\hat{\mathbb{C}}-D_{P}$. Similarly $Q$ has a multiplicity $q-1$ critical point on $L$, a single (simple) critical point elsewhere on $M$, and its other critical points lie in the interior of the 'heart' $\hat{\mathbb{C}}-D_{Q}$.

Since $G l^{P}$ is not a bijection it no longer follows automatically that if $\operatorname{Gal}^{Q}\left(D_{Q}^{\circ}\right)$ is a disc, then its image $\operatorname{Gal}^{P} \circ \operatorname{Gal}^{Q}\left(D_{Q}^{\circ}\right)$ need also be a disc, but this will be the case if the latter contains $p-2$ critical points of $P$ (counted with multiplicity). To apply Theorem 2 we shall require this and the corresponding condition that $G a l^{Q} \circ \operatorname{Gal}^{P}\left(D_{P}^{\circ}\right)$ contain $q-2$ critical points of $Q$. The easiest way to achieve this scenario is to restrict consideration to maps $P$ and $Q$ obtained by pre-composing $P_{0}$ and $Q_{0}$ by Möbius transformations, where

$$
P_{0}(z)=z\left(1+\frac{z}{p-1}\right)^{p-1}
$$


and

$$
Q_{0}(z)=z\left(1+\frac{z}{q-1}\right)^{q-1}
$$

Then $P$ and $Q$ have critical points arranged as in Figure 12, but now inside the 'heart' $\hat{\mathbb{C}}-D_{P}$ we have just one critical point of $P$ (of multiplicity $p-2$ ), and inside the other 'heart' $\widehat{\mathbb{C}}-D_{Q}$ we have just one critical point of $Q$ (of multiplicity $q-2$ ). When these 'interior' critical points lie in $\Lambda_{-}(\mathcal{G}) \cup \Lambda_{+}(\mathcal{G})$ rather than $\Omega(\mathcal{F}, \Delta)$ we have a mating of $C_{p} * C_{q}$ with polynomials $R_{+}$and $R_{-}$of degree $(p-1)(q-1)$, having filled Julia sets homeomorphic to $\Lambda_{+}$and $\Lambda_{-}$respectively.

Comment. In the case $p, q>2$ the correspondence $\operatorname{Gal}^{P}$ is of type $(p-2: p-2)$ when restricted to $\Lambda_{-} \rightarrow \Lambda_{-}$and $(p-1: 1)$ as a map $\Lambda_{-} \rightarrow \Lambda_{+}$. Similarly Gal ${ }^{Q}$ is $(q-2: q-2)$ restricted to $\Lambda_{+} \rightarrow \Lambda_{+}$and $(q-1: 1)$ as a map $\Lambda_{+} \rightarrow \Lambda_{-}$. In the case $p=2$ the $(p-1: 1)$ map becomes a homeomorphism. In the matings described above we observe that $K\left(R_{-}\right)$has a $(q-1)$-fold cover homeomorphic to $K\left(R_{+}\right)$and that $K\left(R_{+}\right)$has a $(p-1)$-fold cover homeomorphic to $K\left(R_{-}\right)$. As we mentioned at the end of Section 3, it is an interesting question as to whether in these circumstances $K\left(R_{+}\right)$is necessarily homeomorphic to $K\left(R_{-}\right)$. In the example below the presence of an additional symmetry provides such a homeomorphism.

Example. Take $p=q=3$. Let

$$
P(z)=\left(\frac{z-1 / 3}{z+1}\right)\left(\frac{z+1 / 3}{z+1}\right)^{2} \text { and } Q(z)=P(-z) .
$$

Then $P$ has a double critical point at $z=-1$ and simple critical points at $z=0$ and $z=-1 / 3$, and $Q$ has a double critical point at $z=+1$ and simple critical points at $z=0$ and $z=+1 / 3$. An elementary calculation shows that Gal $^{P}$ maps $z=-1 / 3$ to $z=+1 / 3$ and $G_{a l}^{Q}$ maps $z=+1 / 3$ back to $z=-1 / 3$. It follows that in this example $\operatorname{Cov}^{P} * \operatorname{Cov}^{Q}$ is a mating of $C_{3} * C_{3}$ with $z \rightarrow z^{4}$. In fact this example has an identical limit set to that obtained from the mating of $C_{3} * C_{2}$ with $z \rightarrow z^{2}$ obtained by setting $a=5$ in the family [1]

$$
z \rightarrow w \text { where }\left(\frac{a z+1}{z+1}\right)^{2}+\left(\frac{a z+1}{z+1}\right)\left(\frac{a w-1}{w-1}\right)+\left(\frac{a w-1}{w-1}\right)^{2}=3,
$$

the mating above of $C_{3} * C_{3}$ with $z \rightarrow z^{4}$ being an index two subcorrespondence of the mating [1] of $C_{3} * C_{2}$ with $z \rightarrow z^{2}$. More generally, given any rational maps $P$ of degree 2 and $Q$ of degree $q$ such that $\operatorname{Cov}^{P} * \operatorname{Cov}^{Q}$ is a mating of $C_{2} * C_{q}$ with a polynomial $R$ of degree $q-1$, if we let $\sigma$ denote the involution $G{ }^{P}$ and $S$ denote the rational function $S(z)=Q(\sigma z)$, then $\operatorname{Cov}^{S} * \operatorname{Cov}^{Q}$ is an index two subcorrespondence of $\operatorname{Cov}^{P} * \operatorname{Cov}^{Q}$ and is a mating of an index two subgroup $C_{q} * C_{q}$ of $C_{2} * C_{q}$ with the degree $(q-1)^{2}$ polynomial $R \circ R$.

\section{Separable correspondences}

An $(r: r)$ correspondence $f: z \rightarrow w$ on the Riemann sphere is called separable if it can be defined by a relation of the form $Q_{+}(z)=Q_{-}(w)$ (where $Q_{+}$and $Q_{-}$are rational functions), and reversible if there exists an involution $\sigma$ such that $z \rightarrow w$ if and only if $\sigma w \rightarrow \sigma z[3$. If $f$ is both separable and reversible the reversing involution $\sigma$ conjugates $\operatorname{Cov}^{Q_{+}}$to $\operatorname{Cov}^{Q-}$ and therefore descends to an involution $\underline{\sigma}$ such that $Q_{-}=\underline{\sigma} Q_{+} \sigma$. Thus, writing $R$ for $Q_{+}$to simplify notation, $f: z \rightarrow w$ 


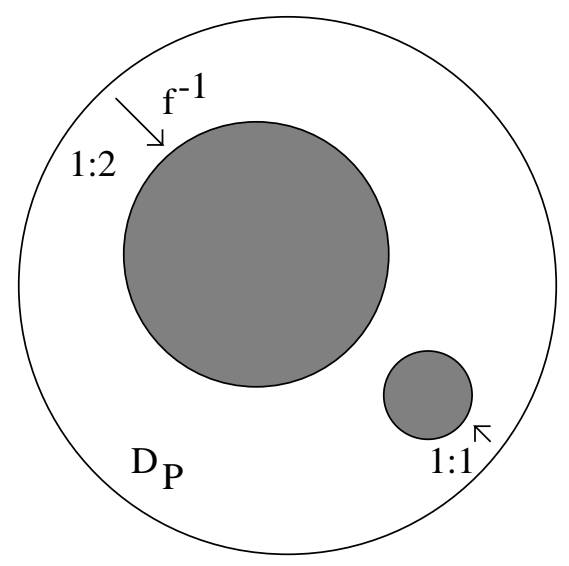

Figure 13.

is reversible separable if and only if there exist a rational map $R$ and involutions $\sigma, \underline{\sigma}$ such that

$$
z \rightarrow w \Leftrightarrow \underline{\sigma} R(z)=R(\sigma w)
$$

Given such an $(r: r)$ correspondence $f$, consider the degree $2 r$ rational map $Q=S$ 。 $R$ obtained by composing with $R$ a rational map $S$ of degree two having $G l^{S}=\underline{\sigma}$, and let $P$ be a rational map of degree two having $G a l^{P}=\sigma$. Now $\mathcal{G}=G a l^{P} \circ G a l^{Q}$ is the union of $f$ and the $(r-1: r-1)$ correspondence $G_{a l}{ }^{P} \circ G a l^{R}$.

Suppose that we can find transversals $D_{P}, D_{Q}$ which have interiors $D_{P}^{\circ}, D_{Q}^{\circ}$ with $\overline{D_{P}^{\circ}}=\overline{D_{P}}$ and $\overline{D_{Q}^{\circ}}=\overline{D_{Q}}$, and such that $D_{P}^{\circ}$ and $D_{Q}^{\circ}$ together cover the Riemann sphere $\hat{\mathbb{C}}$. Then, by Theorem $2, \hat{\mathbb{C}}$ is partitioned into a regular set $\Omega(\mathcal{F}, \Delta)$ and its complement $\Lambda_{-} \sqcup \Lambda_{+}$, where $\Lambda_{-}=\bigcap \mathcal{G}^{-n}\left(D_{P}\right)$ and $\Lambda_{+}=\bigcap \mathcal{G}^{n}\left(D_{Q}\right)$. Consider $\mathcal{G}^{-1}: D_{P}^{\circ} \rightarrow D_{P}^{\circ}$. The image $\mathcal{G}^{-1}\left(D_{P}^{\circ}\right)$ is the disjoint union of $f^{-1}\left(D_{P}^{\circ}\right)$ and $\left(\mathrm{Gal}^{P} \circ \mathrm{Gal}^{R}\right)^{-1}\left(D_{P}^{\circ}\right)\left(\right.$ since $\mathcal{G}^{-1}$ is a $(1: 2 r-1)$ correspondence on $\left.D_{P}^{\circ}\right)$. If each of these sets is a topological disc, then $\mathcal{G}$ is a generalised polynomial-like mapping in an appropriate sense and we can obtain a description of $\Lambda_{-}$and $\Lambda_{+}$. For example with $r=2$, if $f^{-1}\left(D_{P}^{\circ}\right)$ is a disc the correspondence $\mathcal{G}^{-1}$ restricted to $D_{P}^{\circ}$ decomposes as indicated in Figure 13. Now $\cap f^{-n}\left(D_{P}\right)$ (the limit set under unidirectional iteration of $f^{-1}$ ) is homeomorphic to a filled quadratic Julia set, and $\bigcap \mathcal{G}^{-n}\left(D_{P}\right) \sqcup \bigcap \mathcal{G}^{n}\left(D_{Q}\right)$, which is also the global limit set of $f$ under mixed forward and backward iteration, is the closure of a countable infinite union of copies of this quadratic Julia set. See [2, 3] for examples.

We remark that the perturbations of circle-packing representations of $C_{2} * C_{4}$ discussed in [4] fit into the same framework. These $(2: 2)$ reversible separable correspondences $f$ can be regarded as subcorrespondences of $(3: 3)$ correspondences of the form $G a l^{P} \circ G a l^{Q}$ with $Q=S \circ R$, where $P, R$ and $S$ are degree 2 rational maps, just as above.

The technique outlined in this section for analysing reversible separable correspondences can be applied to separable correspondences in general. Any separable correspondence can be lifted to a reversible separable correspondence on the trivial 
double cover of the sphere (one sheet for forward iteration of the correspondence, and one for backward iteration) [3]. See [2] for computer plots of examples.

\section{Conclusion}

There is a well-established construction which 'mates' two Fuchsian groups which are isomorphic as abstract groups and have limit sets each the whole of $\mathbb{R} \cup \infty$, to construct a quasifuchsian Kleinian group with limit set a quasi-circle. Another wellestablished construction is to 'mate' two polynomial maps by fitting together copies of their actions on their filled Julia sets to construct an action of a rational map. The examples above are an indication that a more general kind of construction is possible, involving 'xenotransplants' between the two best known species of onedimensional holomorphic dynamical system - rational maps and Kleinian groups. The key tool above is Klein's combination theorem in its most elementary form. More complicated arrangements involving the same basic building blocks should be possible by considering more sophisticated forms of the Maskit-Klein combination theorems and covering correspondences of rational functions $P$ and $Q$ which are built up as compositions of rational functions of lower degrees in different ways.

\section{REFERENCES}

[1] S. Bullett and C. Penrose, Mating quadratic maps with the modular group, Inventiones Mathematicae 115 (1994), 483-511. MR 95c:58148

[2] S. Bullett and C. Penrose, A gallery of iterated correspondences, Experimental Mathematics 3 (1994), 85-105. MR 96a:58156

[3] S. Bullett and C. Penrose, Regular and limit sets for holomorphic correspondences, QMW preprint 1999.

[4] S. Bullett and C. Penrose, Perturbing circle-packing Kleinian groups as correspondences, Nonlinearity 12 (1999), 635-672. MR 2000d:37054

[5] S. Bullett and W. Harvey, Mating quadratic maps with Kleinian groups via quasiconformal surgery, Electron. Res. Announc. Amer. Math. Soc. 6 (2000), 21-30.

[6] A. Douady and J. Hubbard, On the dynamics of polynomial-like mappings, Ann. Sci. École Norm. Sup. 18 (1985), 287-343. MR 87f:58083

[7] F. Klein, Neue Beiträge zur Riemann'schen Functiontheorie, Math. Ann. 21 (1883), 141-218.

[8] B. Maskit, On Klein's combination theorem, Trans. Amer. Math. Soc. 120 (1965), 499-509. MR 33:274

[9] B. Maskit, On Klein's combination theorem. II, Trans. Amer. Math. Soc. 131 (1968), 32-39. MR 36:6618

[10] B. Maskit, On Klein's combination theorem. III, Advances in the Theory of Riemann Surfaces, Ann. of Math. Studies 66, Princeton University Press, 1971, pp. 297-316. MR 44:6955

[11] B. Maskit, On Klein's combination theorem. IV, Trans. Amer. Math. Soc. 336 (1993), 265294. MR 93e:30088

School of Mathematical Sciences, Queen Mary and Westfield College, University of London, Mile End RoAd, London E1 4NS, United Kingdom

E-mail address: s.r.bullett@qmw.ac.uk 